US. Department of tise Jifterive

U.S. Geplogical Suryey

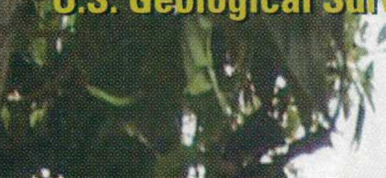

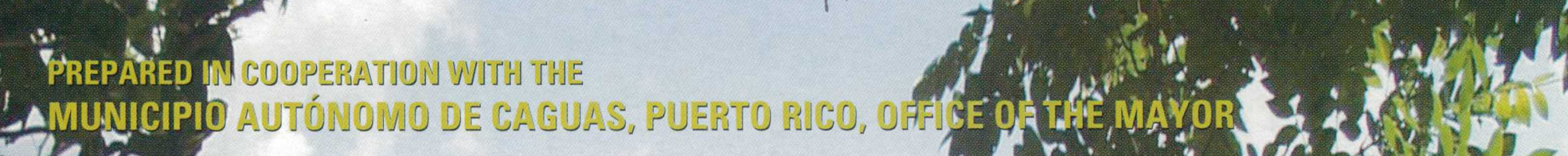

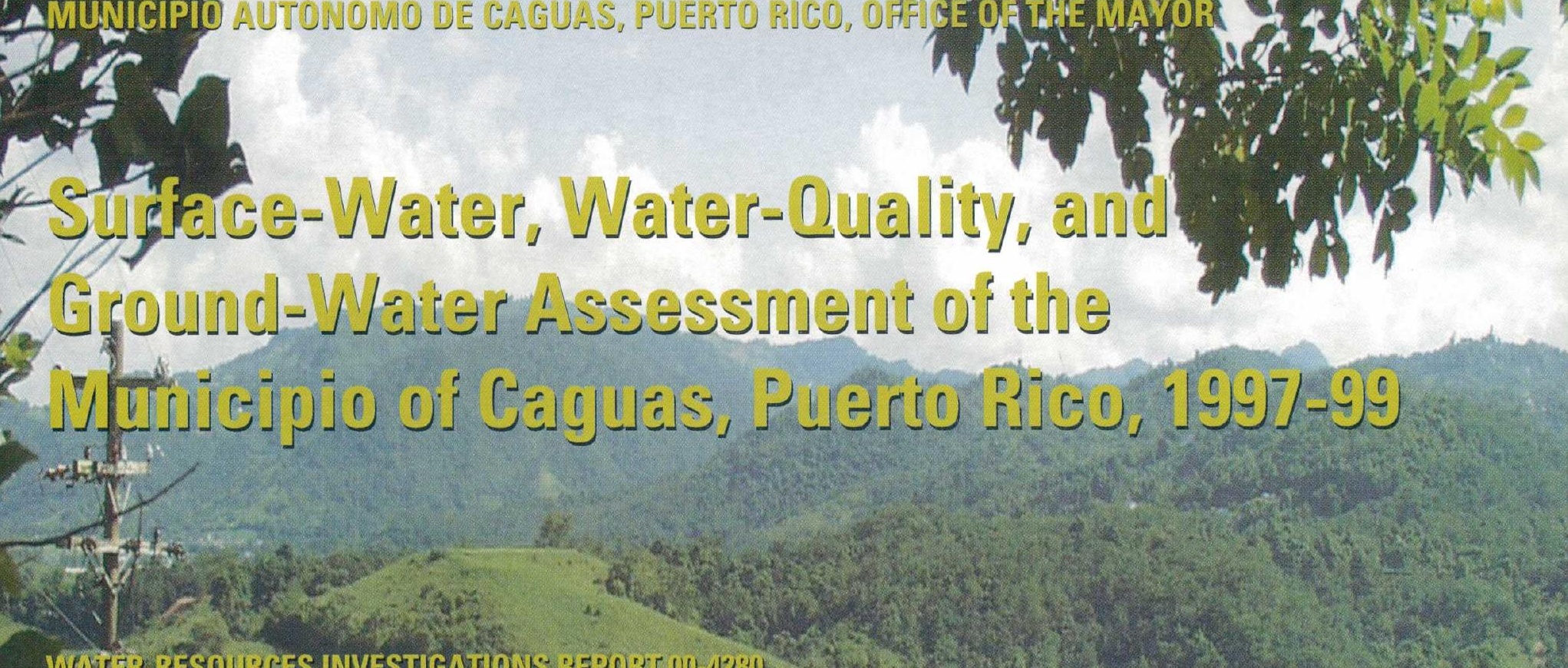

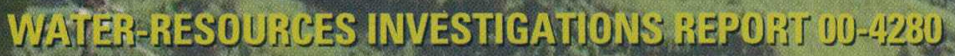

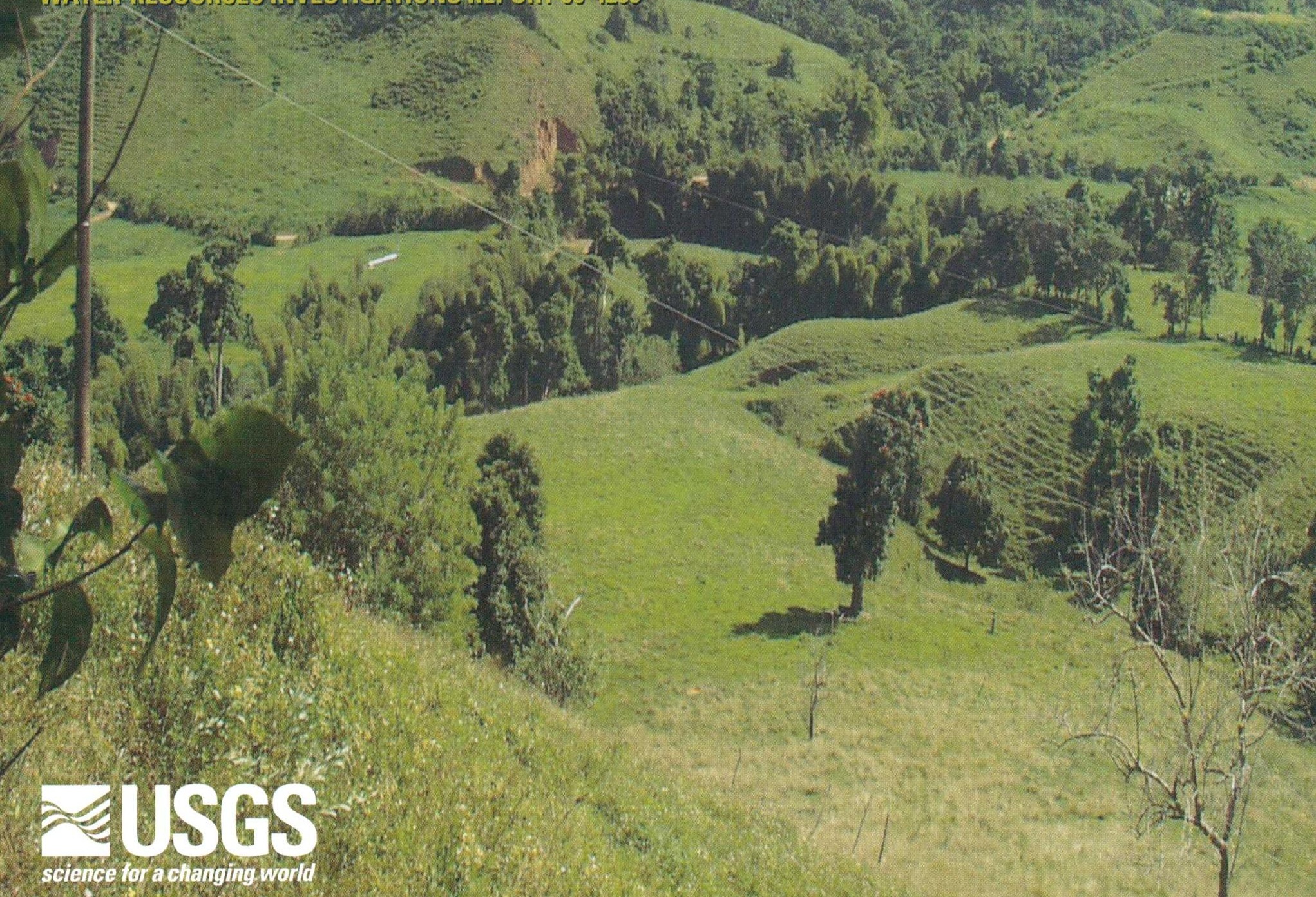




\section{Cover photograph}

View from Highway 1 looking southeast and showing the relatively undeveloped basin of Quebrada Beatriz in :Barrio : Beatriz of the municipio of Caguas. The landslide scar in the foreground marks the approximate location of the stream low-flow and bacteriological quality-of-water station 50053200 at Quebrada Beatriz. The upper watershed of Río Turabo in Barrio San Salvador is in the background. Photograph taken by Jesús Rodríguez-Martínez on December 5, 2000. 


\section{Surface-Water, Water-Quality, and Ground-Water Assessment of the Municipio of Caguas, Puerto Rico, 1997-99}

By Fernando Gómez-Gómez, Jesús Rodríguez-Martínez, Luis Santiago-Rivera, Mario L. Oliveras-Feliciano, and Carlos Conde-Costas

Water-Resources Investigations Report 00-4280

Prepared in cooperation with the MUNICIPIO AUTÓNOMO DE CAGUAS 


\section{U.S. DEPARTMENT OF THE INTERIOR \\ GALE NORTON, Secretary}

\section{U.S. GEOLOGICAL SURVEY}

Charles G. Groat, Director

Use of trade names in this report is for identification purposes only and does not imply endorsement by the U.S. Government.

For additional information write to:

Copies of this report can be purchased from:

District Chief

U.S. Geological Survey

GSA Center, Suite 400-15

651 Federal Drive

Guaynabo, Puerto Rico 00965

U.S. Geological Survey

Branch of Information Services

Box 25286

Denver, CO 80225 


\section{CONTENTS}

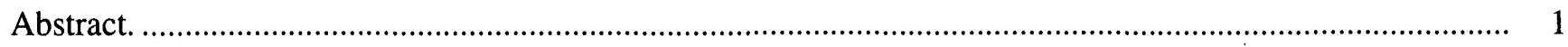

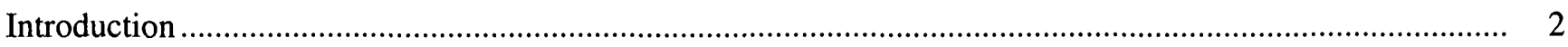

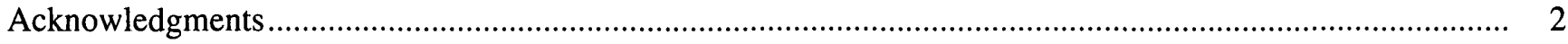

Chapter A: Surface-Water Resources Assessment of the Municipio of Caguas, Puerto Rico, 1997-99

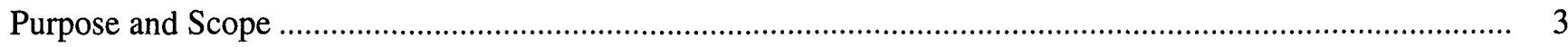

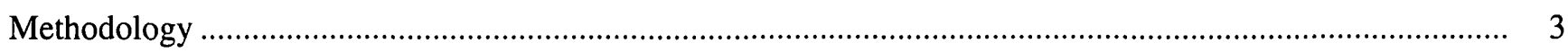

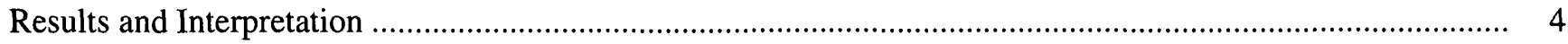

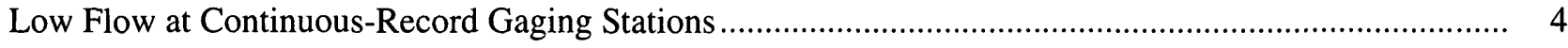

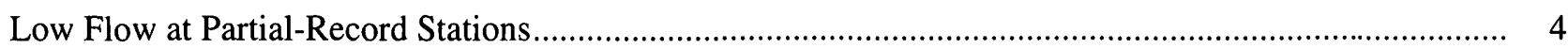

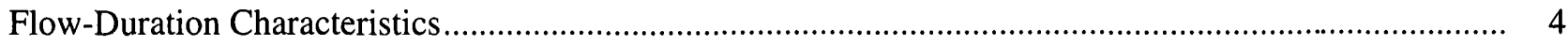

Drainage-Basin Area/Discharge Relation................................................................................... 5

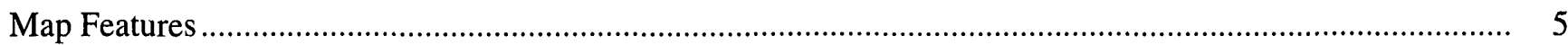

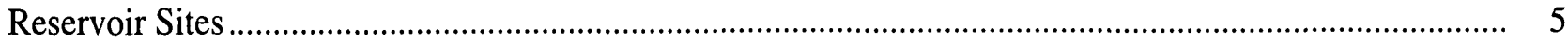

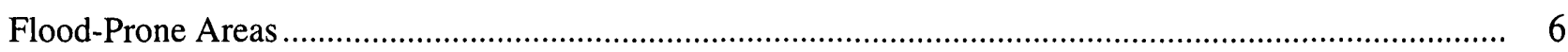

Public Water-Supply Filtration Plants and Waste-Water Treatment Facilities ...................................... 6

Chapter B: Sanitary Quality of Surface Water During Base-Flow Conditions in the Municipio of Caguas, Puerto Rico, 1998-99

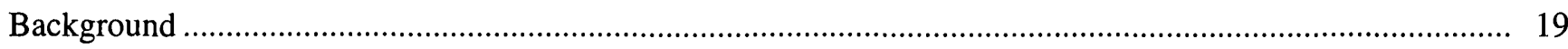

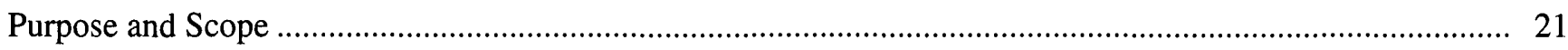

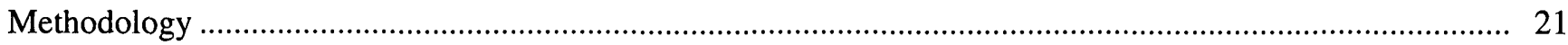

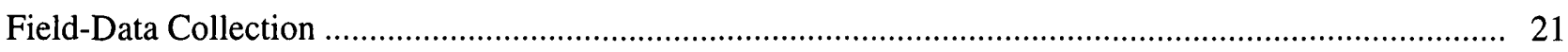

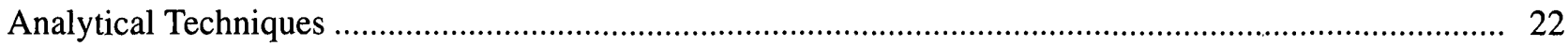

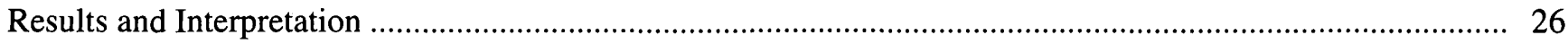

Chapter C: The Hydrogeologic Terranes and Ground-Water Resources in the Municipio of Caguas, Puerto Rico, 1997-99

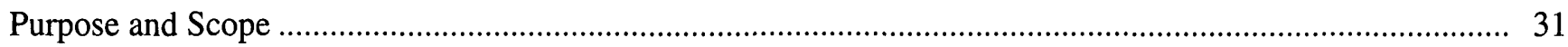

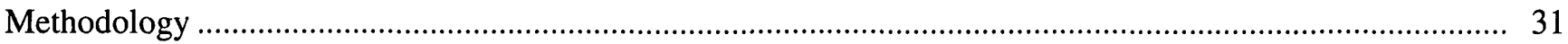

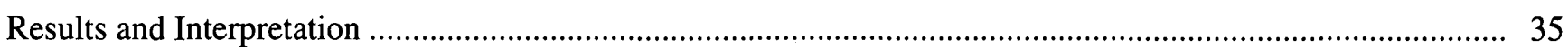

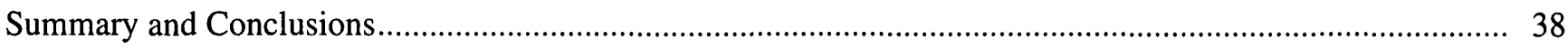

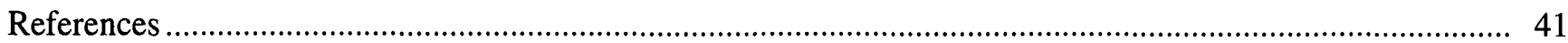




\section{PLATES}

[Plates are in pocket]

1. Streamflow and bacteriological data collection sites, important hydrologic features, and sanitary classification of streams with drainage to or within the municipio of Caguas, Puerto Rico

2. Hydrogeologic terranes, selected sub-basins, potentiometric surface of the alluvial aquifer, lineament traces, and well locations within the municipio of Caguas, Puerto Rico

\section{FIGURES}

1. Map showing location of the municipio of Caguas in relation to the other municipios of Puerto Rico. plates 1 and 2

2. Graph showing long-term geometric mean concentration of fecal coliform bacteria at:

a. the Río Grande de Loíza at Highway PR-189 (USGS station 50055000), November 1984 to

September 1998 .plate 1

b. the Río Cagüitas at Highway PR-30 (USGS station 50055250), October 1984 to December 1998 ......plate 1

c. the Río Bairoa at Highway PR-1 (USGS station 50055400), October 1984 to December 1998. plate 1

d. the Lago Loíza Reservoir (USGS station 50057500), December 1984 to November 1998 plate 1

3. Generalized hydrogeologic section A-A' along the alluvial valley in the municipio of Caguas, Puerto Rico. .plate 2

\section{TABLES}

1. Summary of drainage-basin characteristics, and low flow and flow duration for continuous-record gaging stations within the municipio of Caguas, Puerto Rico

2. Summary of drainage-basin characteristics, and low-flow and flow-duration estimates for partial-record stations within the municipio of Caguas, Puerto Rico

3. Principal features of public water-supply filtration plants, surface-water intakes, public waste-water treatment facilities, and effluent-discharge points.....

4. Ranges of fecal indicator bacteria concentrations typically found in contaminated surface water

5. Fecal coliform and fecal streptococcus bacteria concentrations at selected sites in the municipio of Caguas, Puerto Rico.

6. Classification rationale used in ranking the sanitary quality of streams in the Caguas area, Puerto Rico.

7. Summary of the bacteriological analysis, the water-quality ranking determined for sampling stations, and stream miles classified under each ranking

8. Summary of chemical analyses results of water from selected wells in the municipio of Caguas, Puerto Rico.

9. Hydrogeologic features, including areal extent, normalized ground-water flow per square mile, effective recharge, and relation with hydrogeologic terranes, of selected sub-basins in the municipio of Caguas, Puerto Rico 


\begin{tabular}{rll}
\hline Multiply & \multicolumn{1}{c}{ By } & To obtain \\
\hline acre (acre) & $1,233.489$ & square meter \\
acre-foot (acre-ft) & 1,233 & cubic meter \\
cubic foot per second (ft $\left.{ }^{3} / \mathrm{s}\right)$ & 0.02832 & cubic meter per second \\
foot (ft) & 30.4785 & centimeter \\
foot (ft) & 0.3048 & meter \\
foot per day (ft/d) & 0.3045 & meter per day \\
gallon (gal) & 3.785 & liter \\
gallon per day (gal/d) & 0.00378 & cubic meter per day \\
gallon per minute (gal/min) & 0.00378 & cubic meter per minute \\
inch (in.) & 2.540 & centimeter \\
inch (in.) & 25.4 & millimeter \\
mile (mi) & 1.609 & kilometer \\
million gallon per day (Mgal/d) & 0.04381 & cubic meter per second \\
square foot per day (ft $\left.{ }^{2} / \mathrm{d}\right)$ & 0.0929 & square meter per day \\
square mile (mi $\left.{ }^{2}\right)$ & 2.590 & square kilometer \\
\hline
\end{tabular}

Temperature in degrees Celsius $\left({ }^{\circ} \mathrm{C}\right)$ may be converted to degrees Fahrenheit $\left({ }^{\circ} \mathrm{F}\right)$ as follows:

$$
{ }^{\circ} \mathrm{F}=\left({ }^{\circ} \mathrm{C} \times 1.8\right)+32
$$

\section{Abbreviated water-quality units used in this report:}

$\mu \mathrm{g} / \mathrm{L} \quad$ micrograms per liter

$\mu \mathrm{S} / \mathrm{L} \quad$ microsiemens per liter

\section{Acronyms used in this report:}

FEMA Federal Emergency Management Agency

USGS United States Geological Survey

PRASA Puerto Rico Aqueduct and Sewer Authority

USEPA United States Environmental Protection Agency 


\title{
Surface-Water, Water-Quality, and Ground-Water Assessment of the Municipio of Caguas, Puerto Rico, 1997-99
}

\author{
By Fernando Gómez-Gómez, Jesús Rodríguez-Martínez, Luis Santiago-Rivera, \\ Mario L. Oliveras-Feliciano, and Carlos Conde-Costas
}

\begin{abstract}
To meet the increasing need for a safe and adequate supply of water in the municipio of Caguas, an integrated surface-water, water-quality, and ground-water assessment of the area was conducted. The major results of this study and other important hydrologic and water-quality features were compiled in a Geographic Information System and are presented in two 1:30,000-scale map plates to facilitate interpretation and use of the diverse waterresources data.
\end{abstract}

Because the supply of safe drinking water was a critical issue during recent dry periods, the surface-water assessment portion of this study focused on analysis of low-flow characteristics in local streams and rivers. Low-flow characteristics were evaluated at four continuous-record gaging stations, based on graphical curve-fitting techniques and log-Pearson Type III frequency curves. Estimates of low-flow characteristics for 16 partial-record stations were generated using graphical-correlation techniques. Flow-duration characteristics for the partial-record stations were estimated using the relation curves developed from the low-flow study. Stream low-flow statistics document the general hydrology under current land- and water-use conditions.

A sanitary quality survey of streams utilized 38 sample stations to evaluate the sanitary quality of about 137 miles of stream channels. River and stream samples for fecal coliform and fecal streptococcus analyses were collected on two occasions at base-flow conditions to evaluate the sanitary quality of streams. Bacteriological analyses indicate that a significant portion of the stream reaches within the municipio of Caguas may have fecal coliform concentrations above the water-quality goal established by the Puerto Rico Environmental Quality Board (Junta de Calidad Ambiental de Puerto Rico) for inland surface waters. Sources of fecal contamination may include: illegal discharge of sewage to storm-water drains, malfunction of sanitary sewer ejectors, clogged and leaking sewage pipes, septic tank leakage, unfenced livestock, and runoff from livestock pens.

Geologic, topographic, soil, hydrogeologic, and streamflow data were used to divide the municipio of Caguas into four hydrogeologic terranes. The integrated database was then used to evaluate the ground-water potential of each hydrogeologic terrane. Analysis suggests that areas with slopes greater than 15 degrees have relatively low ground-water development potential. Fractures may be locally important in enhancing the water-bearing properties in the hydrogeologic terranes containing igneous rocks. Potentiometric-surface elevations from wells were compiled and collected during this study to characterize ground-water flow in the four hydrogeologic terranes. The resultant potentiometric-surface map indicates that the alluvial aquifer and streams in the lowland parts of the municipio of Caguas are hydraulically connected. 
The integrated hydrogeologic approach used in this study can serve as an important tool for regulatory agencies of Puerto Rico and the municipio of Caguas to evaluate the ground-water resource development potential, examine groundand surface-water interaction, and determine the effect of land-use practices on ground-water quantity and quality in the municipio of Caguas.

Stream low-flow statistics document the general hydrology under current land and water use. Low-flow statistics may substantially change as a result of streamflow diversions for public supply, increase in ground-water development, waste-water discharges, and flood-control measures; this current analysis provides baseline information to evaluate these impacts and develop water budgets.

\section{INTRODUCTION}

The municipio of Caguas, with an estimated population of 141,871 in 1997 (U.S. Department of Commerce, 1998a) and a land area of 58.9 square miles $\left(\mathrm{mi}^{2}\right)$, has inadequate public water supplies during extended periods of normal to below normal rainfall (fig. 1, see plate 1). The deficiency in public water supply became critical in 1994 , as a result of the nearly 30-percent-below-normal rainfall that occurred for the period August 1993 (first month with a significant rainfall deficiency) to September 1994 (when floods occurred in the area). The impact of reduced streamflow affected much of northern Puerto Rico, but was severe in the Caguas area where the public water-supply demand is about 18 million gallons per day (Mgal/d); only about $8 \mathrm{Mgal} / \mathrm{d}$ were available from surface- and ground-water sources within the municipio during average rainfall years prior to the drought (Wanda Molina, USGS, written commun., 1997).

The greater Caguas urban area lies at the end of the "water-supply distribution pipeline" from filtration plants in adjacent municipios. During the latter months of the summer of 1994, public water-supply transfers to Caguas were suspended for all practical purposes because the limited supply was consumed by the communities connected to the same pipeline systems, but closer to the filtration plants (fig. 1). This led to a crisis in the availability of potable water increasing the deficit of the supply from an average of about $3 \mathrm{Mgal} / \mathrm{d}$, which existed prior to the low rainfall period, to about $14 \mathrm{Mgal} / \mathrm{d}$ during the summer of 1994.

To ensure an adequate supply of safe drinking water, the municipio of Caguas requested the U.S. Geological Survey (USGS) to conduct a comprehensive surface- and ground-water resource assessment, and water-quality analysis of streams. This information will be an integral part of the territorial development plan being developed by the municipio of Caguas, that will take into consideration the sustainable use of water resources.

To facilitate this effort, thematic maps were developed to delineate the hydrologic and stream bacteriological (sanitary) conditions, and to define the water-bearing properties of major rock units. A description of the methods and techniques used to conduct the analyses and interpretations made are given in separate chapters of this report. Chapter A documents the results of the surface-water assessment, Chapter B documents stream bacteriological conditions, and Chapter $\mathrm{C}$ documents ground-water availability.

\section{ACKNOWLEDGMENTS}

The authors acknowledge the Honorable William Miranda Marín, Mayor of the Municipio Autónomo de Caguas, and Engineer Ricardo Echevarría Negrón, Director of the Departamento de Planificacíon y Permisos, for their awareness of the need for this assessment to help develop land-use strategies by municipal authorities to promote the sustainable use of water resources. The authors thank Marilyn Santiago and Betzaida Reyes from the USGS Caribbean District's Geographic Information System Unit, for the preparation of the spatial databases and maps presented in this report, and Francisco Maldonado, USGS Caribbean District Scientific Illustrator, for the final compilation and editing of the plates. 


\section{CHAPTER A:}

\section{Surface-Water Resources Assessment of the Municipio of Caguas, Puerto Rico, 1997-99}

\section{By Luis Santiago-Rivera}

\section{PURPOSE AND SCOPE}

The USGS in cooperation with the Municipio Autónomo de Caguas conducted an investigation of the surface-water resources from October 1, 1997, to September 30, 1999, in the area encompassed primarily by the geographic limits of the municipio of Caguas. A major component of the study consisted of an assessment of the magnitude and frequency of low-flow and flow-duration characteristics, which are important for storage-facility design, waste-load allocation, water-supply planning, recreation, and conservation of wildlife. In addition, the current assessment provides reference conditions to assess future changes in flow magnitude, duration, and frequency.

The low-flow and flow-duration network in Caguas includes 4 long-term continuous-record gaging stations and 16 partial-record stations. Of the 4 continuous monitoring stations, 1 is located along Río Grande de Loíza, 2 along Río Cagüitas, and 1 along Río Turabo, in which Ríos Cagüitas and Turabo are second-order tributaries of Río Grande de Loíza. The 16 partial-record stations are distributed among a number of first- to fourth-order streams in the Río Grande de Loíza drainage basin (plate 1). Streamflow was measured concurrently at least eight times at the index gaging stations and partial-record stations at selected base-flow recessions during a 1-year period to obtain low-flow and flow-duration estimates. The 7-day, 10-year $\left(7 \mathrm{Q}_{10}\right)$ and the 7-day, 2-year $\left(7 \mathrm{Q}_{2}\right)$ low-flow frequency characteristics were computed for the continuous-record gaging stations and estimated for the partial-record stations. Flow-duration characteristics for 90-, 95-, and 99-percent probability of exceedance are included for the 4 continuous-record gaging stations and 16 partial-record stations.

Pertinent information regarding surface-water hydrology within the municipio of Caguas is presented on a thematic map (plate 1). The map displays the following information:

- stream discharge data-collection sites;

- drainage basins for the streamflow sites in which low flows were determined;

- existing and potential reservoir sites (Black and Veatch, 1976);

- flood-prone areas as delineated by the Federal Emergency Management Agency (FEMA, 1996);

- points of withdrawal of public water supply;

- water filtration plants; and

- points of return flow of waste-water treatment facilities.

This information can be used by planners, water managers, and the general public to promote the sustainable use of water resources.

\section{METHODOLOGY}

A series of 8 discharge measurements were taken concurrently at the 4 continuous- and the 16 partial-record stream-gaging stations to provide a basis for a systematic low-flow and flow-duration analysis. A number of techniques were then applied to compute low-flow characteristics at continuous- and partial-record stations. Analyses of low-flow characteristics for continuous-record gaging stations 
(index stations) were based on frequency analyses of the annual minimum 7-day low flows (table 1). Using the streamflow data generated during this study, the partial-record station base-flow measurements were related to concurrent base-flow discharge measurements or daily mean flows at nearby index stations (Riggs, 1972). The low-flow characteristics at partial-record stations were then estimated using the corresponding characteristics at the index station (table 2).

Flow-duration characteristics were computed for the index stations (Searcy, 1959), and flowduration characteristics were estimated for the partialrecord stations using flow-duration characteristics of the index station in conjunction with the relation curve previously developed by correlation methods for the low-flow study. All low-flow and flow-duration characteristics for index stations and partial-record stations were calculated without incorporating the effects of public water-supply withdrawals and/or waste-water discharges upstream of stations. Estimated withdrawals and effluent discharges, however, were compiled from data furnished by the Puerto Rico Aqueduct and Sewer Authority (PRASA) and reported by Black and Veatch (1996), and are presented in the header of each recording station in tables 1 and 2.

\section{RESULTS AND INTERPRETATION}

\section{Low Flow at Continuous-Record Gaging Stations}

A continuous-record gaging station (index station) is a station where daily flow data are systematically collected over a period of years. Lowflow frequency curves were derived for four index stations using the method described by Riggs (1972) and by adaptation of the log-Pearson Type III floodfrequency program described by the Interagency Advisory Committee on Water Data (1982). The $7 \mathrm{Q}_{10}$ and the $7 \mathrm{Q}_{2}$ low-flow frequency characteristics computed for the index stations are presented in table 1 . Index stations used in this analysis are affected by public water-supply withdrawals and/or wastewater treatment facility discharges. However, this may only be significant at the Río Grande de Loíza at Caguas gaging station, where low flows may have decreased by about 5.6 cubic feet per second $\left(\mathrm{ft}^{3} / \mathrm{s}\right)$ (Wanda Molina, USGS, written commun., 2000). Capture of streamflow upstream of this station can result in computational underestimates of low-flow statistics at partial-record stations, for which gaging station 50055000 was used as the index station.

\section{Low Flow at Partial-Record Stations}

A partial-record station is a site where streamflow and/or water-quality data are collected systematically over a limited period of time for use in hydrologic analyses. At these stations, sufficient baseflow measurements are made to define an adequate relation with concurrent flows at a nearby index station. Low-flow characteristics for partial-record stations were estimated using a graphical correlation technique (Riggs, 1972). This technique relates baseflow discharge measurements made at partial-record stations with concurrent discharges measured at the index stations. This estimating technique transfers low-flow characteristics computed by the log-Pearson Type III frequency distribution for the index stations to the graphically-determined relation curve to determine the corresponding low-flow characteristics at the partial-record stations. Low-flow characteristics were estimated for 16 partial-record stations and are presented in table 2. Partial-record stations were located as near as possible to corresponding index stations; the partial- and continuous-recording stations have similar drainage basin size and geologic settings. At these stations, streamflow was measured concurrently at least eight times at different base-flow recessions from February 1998 to April 1999. Instantaneous discharge measurements made at partial-record stations are given in Díaz and others (1998).

\section{Flow-Duration Characteristics}

Flow-duration characteristics were computed for four index stations using techniques developed by Searcy (1959). The analyses of the index stations were based on daily discharge records for complete water 
years (October 1 to September 30), and the results are presented in table 1. Flow-duration characteristics were estimated for 16 partial-record stations using flow-duration characteristics derived for the index stations in conjunction with the relation curves developed for the low-flow study. Index station discharges for the 90-, 95-, and 99-percent flow duration were used as the independent variable in the relation curve to estimate the discharges for the corresponding percent-duration points at the 16 partial-record stations (table 2).

\section{Drainage-Basin Area/Discharge Relation}

Using the drainage-basin size contributing to each monitoring station and the low-flow statistics, a preliminary analysis of discharge yield per unitdrainage area was conducted. This analysis provides a means to evaluate effective recharge within the study area. The Río Turabo at Barrio San Salvador (station 50052925) and the Quebrada Maracai at Barrio San Salvador (station 50052950) drainage basins have a 99-percentile discharge yield per unit area of 0.93 and 0.9 cubic feet per second per square mile $\left(\mathrm{ft}^{3} / \mathrm{s}-\mathrm{mi}^{2}\right)$, respectively. The surface-water contribution of these two basins is $3.4 \mathrm{ft}^{3} / \mathrm{s}$, based on the 99-percent discharge exceedance, which is 16 percent of the discharge registered at Río Grande de Loíza station (50055000), but only 8 percent of the contributing drainage area. Discharge yields of the Río Turabo and Quebrada Maracai drainage basins are significantly higher than those of the other drainage basins within the municipio of Caguas (tables 1, 2, and plate 1). These other stations have an average 99-percentile discharge per unit area of $0.18 \mathrm{ft}^{3} / \mathrm{s}-\mathrm{mi}^{2}$ and range from $0.04 \mathrm{ft}^{3} / \mathrm{s}-\mathrm{mi}^{2}$ at Quebrada Naranjito at Barrio Borinquen (station 50053080) and at Río Cagüitas at Highway 156 near Caguas (station 50055120 ) to $0.59 \mathrm{ft}^{3} / \mathrm{s}-\mathrm{mi}^{2}$ at Río Turabo above Borinquen (station 50053025).

\section{MAP FEATURES}

A 1:30,000-scale map (plate 1) was developed to show the location of hydrologic data-collection stations, drainage basins, the 100- and 500-year flood-prone areas delineated by FEMA (1996) as the 1.0 and 0.2 percent chance of flooding in any given year, and existing or potential reservoir sites (Black and Veatch, 1976). The thematic map also summarizes the hydrologic monitoring-station characteristics and stream bacteriological (sanitary) quality during lowflow conditions (see Chapter B for discussion of water quality).

\section{Reservoir Sites}

One existing reservoir and two potential reservoir sites are shown in plate 1 . The Lago Loíza reservoir located in the Río Grande de Loíza basin with a drainage area of $208 \mathrm{mi}^{2}$ is the major source of public water supply for the San Juan metropolitan area. This reservoir had an initial storage capacity of 21,700 acre-feet (acre-ft) when built in 1953, but had only 11,500 acre-ft in 1994 (Webb and Soler-López, 1997), which indicates a long-term sedimentation rate of 249 acre-ft per year. The loss in storage resulted in a loss of the reservoir's firm yield from about $70 \mathrm{Mgal} / \mathrm{d}$ to about $35 \mathrm{Mgal} / \mathrm{d}$. Dredging of the reservoir was conducted during 1998 and 1999 to increase storage by about 5,000 acre-ft, which theoretically increased the firm yield from 35 to $50 \mathrm{Mgal} / \mathrm{d}$. The firm yield, however, may be substantially lower as a result of the increasing trend of streamflow diversions usptream from the reservoir for public water supply.

Quebrada Beatriz and Quebrada de las Quebradillas are the two potential reservoir sites, which are identified in Black and Veatch (1976). The potential reservoir site on Quebrada Beatriz, with a drainage area of approximately $4.40 \mathrm{mi}^{2}$ and a potential storage of about 6,120 acre-ft, would have a firm yield of approximately $5.5 \mathrm{Mgal} / \mathrm{d}$. The potential reservoir site on the Quebrada de las Quebradillas, with a drainage area of approximately $6.3 \mathrm{mi}^{2}$ and a potential storage of about 6,540 acre- $\mathrm{ft}$, would have a firm yield of approximately $5.8 \mathrm{Mgal} / \mathrm{d}$ (Black and Veatch, 1976). A surface-water intake is presently operating within the Quebrada de las Quebradillas proposed reservoir site to supply PRASA's Quebrada de las Quebradillas filtration plant. 


\section{Flood-Prone Areas}

FEMA presents three different types of floodprone areas for the city of Caguas (FEMA, 1996). These areas are shown on plate 1 as Zone A, the area inundated by the 100-year flood (the annual flood that has a 1 in 100 probability of recurrence) for which no Base Flood Elevations (BFEs) have been determined; Zone AE, the area inundated by the 100 -year flood, for which BFEs have been determined; and Zone X500, the area inundated by the 500 -year flood, equivalent to a 0.2 percent annual chance of flooding; an area inundated by a 1 percent annual chance of flooding with average depths of less than 1 foot $(\mathrm{ft})$ or with a drainage area less than $1 \mathrm{mi}^{2}$, or an area protected by levees from a 1 percent annual chance of flooding.

\section{Public Water-Supply Filtration Plants and Waste-Water Treatment Facilities}

There are four public water-supply filtration plants within the municipio of Caguas (plate 1). These are the Quebrada de las Quebradillas filtration plant (also referred to as Caguas filtration plant) with intakes at Quebrada de las Quebradillas (gravity intake) and Río Turabo (pump station); the Caguas Norte filtration facility with an intake at Río Grande de Loíza (pump station); the San Salvador filtration plant with gravity intake from Quebrada Morena; and the San Antonio filtration plant with an intake directly from Lago Loíza reservoir or from an infiltration gallery near the west bank of the reservoir. Three public waste-water treatment facilities are located in the municipio of Caguas. These are the Caguas regional waste-water treatment facility with discharge to the Río Bairoa; the Las Carolinas waste-water treatment facility with discharge to the Río Cagüitas; and the Borinquen waste-water treatment facility with discharge to the Río Turabo. The location of the facilities and other pertinent data are listed in table 3 and shown on plate 1 . 
Table 1. Summary of drainage-basin characteristics, and low flow and flow duration for continuous-record gaging stations within the municipio of Caguas, Puerto Rico

[lat, latitude; long, longitude; $\mathrm{mi}$, mile; $\mathrm{km}$, kilometer; $\mathrm{mi}^{2}$, square mile; $\mathrm{km}^{2}$, square kilometer; $\mathrm{ft}$, feet; $\mathrm{m}, \mathrm{meter} ; \mathrm{ft}^{3} / \mathrm{s}, \mathrm{cubic}$ feet per second]

\section{RÍO GRANDE DE LOÍZA BASIN}

\section{Rio Turabo above Borinquen, Puerto Rico}

LOCATION--Lat $18^{\circ} 09^{\prime} 35^{\prime \prime}$, long $66^{\circ} 02^{\prime} 26^{\prime \prime}$, Hydrologic Unit 21010005 , on left bank, at Highway $765,1.2 \mathrm{mi}(1.9 \mathrm{~km})$ south of Villa Borinquen, $8.1 \mathrm{mi}(13 \mathrm{~km})$ upstream from Río Grande de Loíza.

DRAINAGE AREA--7.14 $\mathrm{mi}^{2}\left(18 \mathrm{~km}^{2}\right)$.

PERIOD OF RECORD ANALYZED--January 1990 to September 1997.

LOW-FLOW ANALYSIS--Log-Pearson Type III frequency distribution.

REMARKS--A diversion of $0.31 \mathrm{ft}^{3} / \mathrm{s}$ is made upstream of station for public water supply.

LOW-FLOW CHARACTERISTICS

[Based on 1990-97 water years]

\begin{tabular}{cc}
\hline Low-flow characteristics & Discharge $\left(\mathrm{ft}^{3} / \mathrm{s}\right)$ \\
\hline 7-day, 2-year & 5.5 \\
7-day, 10-year & 4.0 \\
\hline
\end{tabular}

FLOW-DURATION CHARACTERISTICS

[Based on 1990-97 water years]

\begin{tabular}{lccc}
\hline \multicolumn{4}{c}{ Discharge $\left(\mathrm{ft}^{3} / \mathrm{s}\right)$ that was equaled or exceeded for indicated percentage of time } \\
\hline Percent & 90 & 95 & 99 \\
Discharge & 5.9 & 5.3 & 4.2 \\
\hline
\end{tabular}

\section{Río Grande de Loíza at Caguas, Puerto Rico}

LOCATION--Lat $18^{\circ} 14^{\prime} 33^{\prime \prime}$, long $66^{\circ} 00^{\prime} 34^{\prime \prime}$, Hydrologic Unit 21010005 , on right bank, $250 \mathrm{ft}(76 \mathrm{~m})$ upstream from bridge on Highway $189,1.2 \mathrm{mi}$ $(1.9 \mathrm{~km})$ downstream from Río Turabo, and $1.8 \mathrm{mi}(2.9 \mathrm{~km})$ east of Caguas plaza.

DRAINAGE AREA--90 $\mathrm{mi}^{2}\left(233 \mathrm{~km}^{2}\right)$.

PERIOD OF RECORD ANALYZED--October 1961 to September 1997.

LOW-FLOW ANALYSIS--Log-Pearson Type III frequency distribution.

REMARKS--A diversion of $6.5 \mathrm{ft}^{3} / \mathrm{s}$ is made upstream of station for public water supply and 0.9 cubic feet per second is recovered from waste-water treatment plant return flow.

LOW-FLOW CHARACTERISTICS

[Based on 1961-97 water years]

\begin{tabular}{cc}
\hline Low-flow characteristics & Discharge $\left(\mathrm{ft}^{3} / \mathrm{s}\right)$ \\
\hline 7-day, 2-year & 31.9 \\
7-day, 10-year & 17.4 \\
\hline
\end{tabular}

FLOW-DURATION CHARACTERISTICS

[Based on 1961-97 water years]

\begin{tabular}{lccc}
\hline \multicolumn{4}{c}{ Discharge $\left(\mathrm{ft}^{3} / \mathrm{s}\right)$ that was equaled or exceeded for indicated percentage of time } \\
\hline Percent & 90 & 95 & 99 \\
Discharge & 39.1 & 30.5 & 21.3 \\
\hline
\end{tabular}


Table 1. Summary of drainage-basin characteristics, and low-flow and flow-duration for continuous-record gaging stations within the municipio of Caguas, Puerto Rico-Continued

\section{Río GRANDE DE LOÍZA BASIN—Continued \\ 50055100 Río Cagüitas near Aguas Buenas, Puerto Rico}

LOCATION--Lat $18^{\circ} 14^{\prime} 48^{\prime \prime}$, long $66^{\circ} 05^{\prime} 37^{\prime \prime}$, Hydrologic Unit 21010005 , on right bank, $450 \mathrm{ft}$ (137 m) upstream from bridge on Highway $777,1.0 \mathrm{mi}$

$(1.6 \mathrm{~km})$ southeast from Aguas Buenas, $3.9 \mathrm{mi}(6.3 \mathrm{~km})$ northwest from Caguas, and $2.1 \mathrm{mi}(3.4 \mathrm{~km})$ southwest from Las Carolinas.

DRAINAGE AREA--5.3 $\mathrm{mi}^{2}\left(14 \mathrm{~km}^{2}\right)$.

PERIOD OF RECORD ANALYZED--February 1990 to September 1997.

LOW-FLOW ANALYSIS--Log-Pearson Type III frequency distribution.

REMARKS--A diversion of $0.2 \mathrm{ft}^{3} / \mathrm{s}$ is made upstream of station for public water supply.

LOW-FLOW CHARACTERISTICS

[Based on 1990-97 water years]

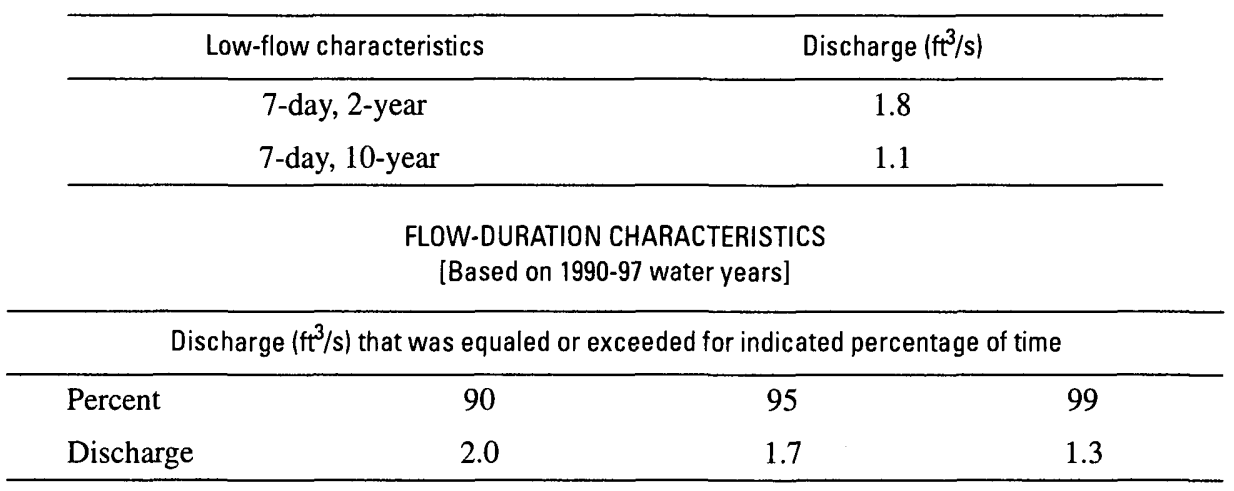

50058350 Río Cañas at Río Cañas, Puerto Rico

LOCATION--Lat $18^{\circ} 17^{\prime} 41^{\prime \prime}$, long $66^{\circ} 02^{\prime} 44^{\prime \prime}$, Hydrologic Unit 21010005 , at right bank, off road 798 , upstream side of bridge on Highway $52,0.5 \mathrm{mi}$ $(0.8 \mathrm{~km})$ northeast from Escuela Segunda Unidad de Francisco Valdés, and $0.8 \mathrm{mi}(1.3 \mathrm{~km})$ north of La Barra.

DRAINAGE AREA-- $7.5 \mathrm{mi}^{2}\left(20 \mathrm{~km}^{2}\right)$.

PERIOD OF RECORD ANALYZED--March 1990 to September 1997.

LOW-FLOW ANALYSIS--Log-Pearson Type III frequency distribution.

REMARKS--None.

LOW-FLOW CHARACTERISTICS

[Based on 1990-97 water years]

\begin{tabular}{cc}
\hline Low-flow characteristics & Discharge $\left(\mathrm{ft}^{3} / \mathrm{s}\right)$ \\
\hline 7-day, 2-year & 2.5 \\
7-day, 10-year & 1.4 \\
\hline
\end{tabular}

FLOW-DURATION CHARACTERISTICS

[Based on 1990-97 water years]

\begin{tabular}{lccc}
\hline \multicolumn{4}{c}{ Discharge $\left(\mathrm{ft}^{3} / \mathrm{s}\right)$ that was equaled or exceeded for indicated percentage of time } \\
\hline Percent & 90 & 95 & 99 \\
Discharge & 2.5 & 2.0 & 1.4 \\
\hline
\end{tabular}


Table 2. Summary of drainage-basin characteristics, and low-flow and flow-duration estimates for partial-record stations within the municipio of Caguas, Puerto Rico

[lat, latitude; long, longitude; $\mathrm{mi}$, mile; $\mathrm{km}$, kilometer; $\mathrm{mi}^{2}$, square mile; $\mathrm{km}^{2}$, square kilometer; $\mathrm{ft}, \mathrm{feet} ; \mathrm{m}$, meter; $\mathrm{ft}^{3} / \mathrm{s}$, cubic feet per second; <, less than]

\section{RÍO GRANDE DE LOÍZA BASIN}

\section{Río Turabo at Barrio San Salvador, Puerto Rico}

LOCATION--Lat $18^{\circ} 08^{\prime} 37^{\prime \prime}$, long $66^{\circ} 03^{\prime} 05^{\prime \prime}$, Hydrologic Unit 21010005, at Barrio San Salvador, $2.7 \mathrm{mi}(4.3 \mathrm{~km})$ southwest of Cerro Gregorio, $0.8 \mathrm{mi}$

$(1.3 \mathrm{~km})$ northwest of Escuela Segunda Unidad de Mercedes Palma, and $2.4 \mathrm{mi}(3.9 \mathrm{~km})$ southwest from Villa Borinquen.

DRAINAGE AREA-- $1.4 \mathrm{mi}^{2}\left(3.4 \mathrm{~km}^{2}\right)$.

LOW-FLOW AND FLOW-DURATION ESTIMATES--Based on correlation of 8 base-flow measurements with concurrent base flows at gaging station 50053025

REMARKS--None.

LOW-FLOW CHARACTERISTICS

\begin{tabular}{|c|c|c|c|}
\hline \multicolumn{2}{|c|}{ Low-flow characteristics } & \multicolumn{2}{|c|}{ Discharge $\left(\mathrm{ft}^{3} / \mathrm{s}\right)$} \\
\hline \multicolumn{2}{|c|}{ 7-day, 2-year } & \multicolumn{2}{|c|}{1.6} \\
\hline \multicolumn{2}{|c|}{ 7-day, 10-year } & \multicolumn{2}{|c|}{1.3} \\
\hline \multicolumn{4}{|c|}{ FLOW-DURATION CHARACTERISTICS } \\
\hline \multicolumn{4}{|c|}{ Discharge $\left(\mathrm{ft}^{3} / \mathrm{s}\right)$ that was equaled or exceeded for indicated percentage of time } \\
\hline Percent & 90 & 95 & 99 \\
\hline Discharge & 1.7 & 1.6 & 1.3 \\
\hline
\end{tabular}

\section{Quebrada Maracai at Barrio San Salvador, Puerto Rico}

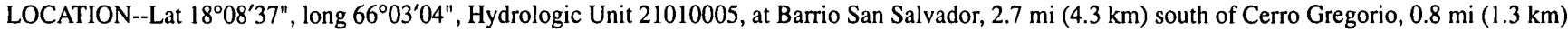
northwest of Escuela Segunda Unidad de Mercedes Palma, and $2.4 \mathrm{mi}(3.9 \mathrm{~km})$ southwest of Villa Borinquen.

DRAINAGE AREA-- $2.3 \mathrm{mi}^{2}\left(6.0 \mathrm{~km}^{2}\right)$.

LOW-FLOW AND FLOW-DURATION ESTIMATES--Based on correlation of 8 base-flow measurements with concurrent base flows at gaging station 50053025 .

REMARKS--A diversion of $0.31 \mathrm{ft}^{3} / \mathrm{s}$ is made upstream of station for public water supply.

LOW-FLOW CHARACTERISTICS

\begin{tabular}{cc}
\hline Low-flow characteristics & Discharge $\left(\mathrm{ft}^{3} / \mathrm{s}\right)$ \\
\hline 7-day, 2-year & 2.6 \\
7-day, 10-year & 2.0 \\
\hline
\end{tabular}

FLOW-DURATION CHARACTERISTICS

\begin{tabular}{lccc}
\hline \multicolumn{4}{c}{ Discharge $\left(\mathrm{ft}^{3} / \mathrm{s}\right)$ that was equaled or exceeded for indicated percentage of time } \\
\hline Percent & 90 & 95 & 99 \\
Discharge & 2.7 & 2.5 & 2.1 \\
\hline
\end{tabular}


Table 2. Summary of drainage-basin characteristics, and low-flow and flow-duration estimates for partial-record stations within the municipio of Caguas, Puerto Rico-Continued

\section{RÍO GRANDE DE LOÍZA BASIN-Continued}

\section{Río Turabo at Highway 765 at Barrio Borinquen, Puerto Rico}

LOCATION--Lat $18^{\circ} 10^{\prime} 45^{\prime \prime}$, long $66^{\circ} 02^{\prime} 33^{\prime \prime}$, Hydrologic Unit 21010005 , at Barrio Borinquen, $2.8 \mathrm{mi}(4.5 \mathrm{~km})$ northwest of Cerro Gregorio, $3.0 \mathrm{mi}(4.8 \mathrm{~km})$ northeast of Cerro Las Piñas, and $1.0 \mathrm{mi}(1.6 \mathrm{~km})$ from El Naranjito.

DRAINAGE AREA--8.1 $\mathrm{mi}^{2}\left(21 \mathrm{~km}^{2}\right)$.

LOW-FLOW AND FLOW-DURATION ESTIMATES--Based on correlation of 8 base-flow measurements with concurrent base flows at gaging station 50053025 .

REMARKS--A diversion of $0.31 \mathrm{ft}^{3} / \mathrm{s}$ is made upstream of station for public water supply.

LOW-FLOW CHARACTERISTICS

\begin{tabular}{cc}
\hline Low-flow characteristics & Discharge $\left(\mathrm{ft}^{3} / \mathrm{s}\right)$ \\
\hline 7-day, 2-year & 5.6 \\
7-day, 10-year & 4.1 \\
\hline
\end{tabular}

FLOW-DURATION CHARACTERISTICS

\begin{tabular}{lccc}
\hline \multicolumn{4}{c}{ Discharge $\left(\mathrm{ft}^{3} / \mathrm{s}\right)$ that was equaled or exceeded for indicated percentage of time } \\
\hline Percent & 90 & 95 & 99 \\
Discharge & 6.0 & 5.4 & 4.2 \\
\hline
\end{tabular}

\section{Quebrada Naranjito at Barrio Borinquen, Puerto Rico}

LOCATION--Lat $18^{\circ} 10^{\prime} 56^{\prime \prime}$, long $66^{\circ} 02^{\prime} 35^{\prime \prime}$, Hydrologic Unit 21010005, at Barrio Borinquen, $3.0 \mathrm{mi}(4.8 \mathrm{~km})$ northwest of Cerro Gregorio, $3.1 \mathrm{mi}(5.0 \mathrm{~km})$ northeast of Cerro Las Piñas, and $1.1 \mathrm{mi}(1.8 \mathrm{~km})$ northwest from El Naranjito.

DRAINAGE AREA--2.2 $\mathrm{mi}^{2}\left(5.8 \mathrm{~km}^{2}\right)$.

LOW-FLOW AND FLOW-DURATION ESTIMATES--Based on correlation of 8 base-flow measurements with concurrent base flows at gaging station 50053025 .

REMARKS--None.

LOW-FLOW CHARACTERISTICS

\begin{tabular}{cc}
\hline Low-flow characteristics & Discharge $\left(\mathrm{ft}^{3} / \mathrm{s}\right)$ \\
\hline 7-day, 2-year & $<0.1$ \\
7-day, 10-year & $<0.1$ \\
\hline
\end{tabular}

FLOW-DURATION CHARACTERISTICS

\begin{tabular}{lccc}
\hline \multicolumn{4}{c}{ Discharge $\left(\mathrm{ft}^{3} / \mathrm{s}\right)$ that was equaled or exceeded for indicated percentage of time } \\
\hline Percent & 90 & 95 & 99 \\
Discharge & $<0.1$ & $<0.1$ & $<0.1$ \\
\hline
\end{tabular}


Table 2. Summary of drainage-basin characteristics, and low-flow and flow-duration estimates for partial-record stations within the municipio of Caguas, Puerto Rico-Continued

\section{RÍO GRANDE DE LOÍZA BASIN-Continued}

\section{Quebrada Sonadora at Barrio Beatriz, Puerto Rico}

LOCATION--Lat $18^{\circ} 10^{\prime} 22^{\prime \prime}$, long $66^{\circ} 03^{\prime} 42^{\prime \prime}$, Hydrologic Unit 21010005 , at Barrio Beatriz, $3.6 \mathrm{mi}(5.8 \mathrm{~km})$ northwest of Cerro Gregorio, $1.8 \mathrm{mi}(2.9 \mathrm{~km})$ northeast of Cerro Las Piñas, and $1.4 \mathrm{mi}(2.2 \mathrm{~km})$ southeast from Villa Borinquen.

DRAINAGE AREA-- $1.2 \mathrm{mi}^{2}\left(3.1 \mathrm{~km}^{2}\right)$.

LOW-FLOW AND FLOW-DURATION ESTIMATES--Based on correlation of 8 base-flow measurements with concurrent base flows at gaging station 50053025 .

REMARKS--None.

LOW-FLOW CHARACTERISTICS

\begin{tabular}{|c|c|c|c|}
\hline \multicolumn{2}{|c|}{ Low-flow characteristics } & \multicolumn{2}{|c|}{ Discharge $\left(\mathrm{ft}^{3} / \mathrm{s}\right)$} \\
\hline \multicolumn{2}{|c|}{ 7-day, 2-year } & \multicolumn{2}{|c|}{0.3} \\
\hline \multicolumn{2}{|c|}{ 7-day, 10-year } & \multicolumn{2}{|c|}{0.2} \\
\hline \multicolumn{4}{|c|}{ FLOW-DURATION CHARACTERISTICS } \\
\hline \multicolumn{4}{|c|}{ Discharge $\left(\mathrm{ft}^{3} / \mathrm{s}\right)$ that was equaled or exceeded for indicated percentage of time } \\
\hline Percent & 90 & 95 & 99 \\
\hline Discharge & 0.3 & 0.3 & 0.2 \\
\hline
\end{tabular}

50053200 Quebrada Beatriz at Barrio Beatriz, Puerto Rico

LOCATION--Lat $18^{\circ} 11^{\prime} 01^{\prime \prime}$, long 6603'52", Hydrologic Unit 21010005 , at Barrio Beatriz, $4.1 \mathrm{mi}(6.6 \mathrm{~km})$ northwest of Cerro Gregorio, $2.3 \mathrm{mi}(3.7 \mathrm{~km})$ northeast of Cerro Las Piñas, and $1.6 \mathrm{mi}(2.6 \mathrm{~km})$ northwest from Villa Borinquen.

DRAINAGE AREA--4.4 $\mathrm{mi}^{2}\left(11 \mathrm{~km}^{2}\right)$.

LOW-FLOW AND FLOW-DURATION ESTIMATES--Based on correlation of 8 base-flow measurements with concurrent base flows at gaging station 50053025.

REMARKS--None.

LOW-FLOW CHARACTERISTICS

\begin{tabular}{cc}
\hline Low-flow characteristics & Discharge $\left(\mathrm{ft}^{3} / \mathrm{s}\right)$ \\
\hline 7-day, 2-year & 0.8 \\
7-day, 10-year & 0.5 \\
\hline
\end{tabular}

FLOW-DURATION CHARACTERISTICS

\begin{tabular}{lccc}
\hline \multicolumn{4}{c}{ Discharge $\left(\mathrm{ft}^{3} / \mathrm{s}\right)$ that was equaled or exceeded for indicated percentage of time } \\
\hline Percent & 90 & 95 & 99 \\
Discharge & 1.0 & 0.8 & 0.5 \\
\hline
\end{tabular}


Table 2. Summary of drainage-basin characteristics, and low-flow and flow-duration estimates for partial-record stations within the municipio of Caguas, Puerto Rico-Continued

\section{RIOO GRANDE DE LOIZZA BASIN—Continued}

\section{Quebrada de las Quebradillas at Barrio Beatriz, Puerto Rico}

LOCATION--Lat $18^{\circ} 11^{\prime} 40^{\prime \prime}$, long 66 $05^{\prime} 25^{\prime \prime}$, Hydrologic Unit 21010005 , at Barrio Beatriz, 2.8 mi (4.5 km) northwest of Cerro Las Piñas, 1.8 mi (2.9 km) northeast of Las Cruces, and $3.4 \mathrm{mi}(5.5 \mathrm{~km})$ northwest from Villa Borinquen.

DRAINAGE AREA--3.8 $\mathrm{mi}^{2}\left(10 \mathrm{~km}^{2}\right)$.

LOW-FLOW AND FLOW-DURATION ESTIMATES--Based on correlation of 8 base-flow measurements with concurrent base flows at gaging station 50053025 .

REMARKS--None.

LOW-FLOW CHARACTERISTICS

\begin{tabular}{cc}
\hline Low-flow characteristics & Discharge $\left(\mathrm{ft}^{3} / \mathrm{s}\right)$ \\
\hline 7-day, 2-year & 1.0 \\
7-day, 10-year & 0.6 \\
\hline
\end{tabular}

FLOW-DURATION CHARACTERISTICS

\begin{tabular}{lccc}
\hline \multicolumn{4}{c}{ Discharge $\left(\mathrm{ft}^{3} / \mathrm{s}\right)$ that was equaled or exceeded for indicated percentage of time } \\
\hline Percent & 90 & 95 & 99 \\
Discharge & 1.1 & 1.0 & 0.7 \\
\hline
\end{tabular}

50053975 Quebrada de las Quebradillas above Dam at Barrio Turabo, Puerto Rico

LOCATION--Lat $18^{\circ} 11^{\prime} 37^{\prime \prime}$, long 66 $04^{\prime} 10^{\prime \prime}$, Hydrologic Unit 21010005 , at Barrio Turabo, $2.8 \mathrm{mi}$ (4.5 km) northeast of Cerro Las Piñas, $2.1 \mathrm{mi}(3.4 \mathrm{~km})$ northeast of Villa Borinquen, and $3.7 \mathrm{mi}(6.0 \mathrm{~km})$ southwest from Caguas plaza.

DRAINAGE AREA-- $6.3 \mathrm{mi}^{2}\left(16 \mathrm{~km}^{2}\right)$

LOW-FLOW AND FLOW-DURATION ESTIMATES--Based on correlation of 8 base-flow measurements with concurrent base flows at gaging station 50053025 .

REMARKS--None.

LOW-FLOW CHARACTERISTICS

\begin{tabular}{cc}
\hline Low-flow characteristics & Discharge $\left(\mathrm{ft}^{3} / \mathrm{s}\right)$ \\
\hline 7-day, 2-year & 1.8 \\
7-day, 10-year & 1.1 \\
\hline
\end{tabular}

FLOW-DURATION CHARACTERISTICS

\begin{tabular}{lccc}
\hline \multicolumn{4}{c}{ Discharge $\left(\mathrm{ft}^{3} / \mathrm{s}\right)$ that was equaled or exceeded for indicated percentage of time } \\
\hline Percent & 90 & 95 & 99 \\
Discharge & 2.0 & 1.7 & 1.2 \\
\hline
\end{tabular}


Table 2. Summary of drainage-basin characteristics, and low-flow and flow-duration estimates for partial-record stations within the municipio of Caguas, Puerto Rico-Continued

\section{RÍO GRANDE DE LOÍZA BASIN-Continued}

\section{Rio Turabo at Villa del Rey near Caguas, Puerto Rico}

LOCATION--Lat $18^{\circ} 12^{\prime} 51^{\prime \prime}$, long $66^{\circ} 02^{\prime} 30^{\prime \prime}$, Hydrologic Unit 21010005 , at Barrio Turabo, $2.5 \mathrm{mi}$ (4.0 km) north of Villa Borinquen, $4.8 \mathrm{mi}$ (7.7 km) northwest of Cerro Gregorio, and $1.6 \mathrm{mi}(2.6 \mathrm{~km})$ southwest from Caguas plaza.

DRAINAGE AREA-- $25 \mathrm{mi}^{2}\left(65 \mathrm{~km}^{2}\right)$.

LOW-FLOW AND FLOW-DURATION ESTIMATES--Based on correlation of 8 base-flow measurements with concurrent base flows at gaging station 50055000 .

REMARKS--A diversion of $0.85 \mathrm{ft}^{3} / \mathrm{s}$ is made upstream of station for public water supply.

LOW-FLOW CHARACTERISTICS

\begin{tabular}{|c|c|c|c|}
\hline \multicolumn{2}{|c|}{ Low-flow characteristics } & \multicolumn{2}{|c|}{ Discharge $\left(\mathrm{ft}^{3} / \mathrm{s}\right)$} \\
\hline \multicolumn{2}{|c|}{ 7-day, 2-year } & \multicolumn{2}{|c|}{3.4} \\
\hline \multicolumn{2}{|c|}{ 7-day, 10-year } & \multicolumn{2}{|c|}{1.5} \\
\hline \multicolumn{4}{|c|}{ FLOW-DURATION CHARACTERISTICS } \\
\hline \multicolumn{4}{|c|}{ Discharge $\left(\mathrm{ft}^{3} / \mathrm{s}\right)$ that was equaled or exceeded for indicated percentage of time } \\
\hline Percent & 90 & 95 & 99 \\
\hline Discharge & 4.6 & 3.2 & 2.0 \\
\hline
\end{tabular}

\section{Río Cagüitas at Highway 156 near Caguas, Puerto Rico}

LOCATION--Lat $18^{\circ} 14^{\prime} 41^{\prime \prime}$, long $66^{\circ} 03^{\prime} 52^{\prime \prime}$, Hydrologic Unit 21010005, at Barrio Cañabón at Highway 156, $3.4 \mathrm{mi}$ (5.5 km) northeast of Los Sumideros, $1.2 \mathrm{mi}(1.9 \mathrm{~km})$ northeast of Escuela Bunker, and $2.0 \mathrm{mi}(3.2 \mathrm{~km})$ northwest from Caguas plaza.

DRAINAGE AREA $-7.4 \mathrm{mi}^{2}\left(19 \mathrm{~km}^{2}\right)$.

LOW-FLOW AND FLOW-DURATION ESTIMATES--Based on correlation of 8 base-flow measurements with concurrent base flows at gaging station 50055100 .

REMARKS--A diversion of $0.2 \mathrm{ft}^{3} / \mathrm{s}$ is made upstream of station for public water supply.

LOW-FLOW CHARACTERISTICS

\begin{tabular}{cc}
\hline Low-flow characteristics & Discharge $\left(\mathrm{ft}^{3} / \mathrm{s}\right)$ \\
\hline 7-day, 2-year & 0.5 \\
\hline 7-day, 10-year & 0.2 \\
\hline
\end{tabular}

FLOW-DURATION CHARACTERISTICS

\begin{tabular}{lccc}
\hline \multicolumn{4}{c}{ Discharge $\left(\mathrm{ft}^{3} / \mathrm{s}\right)$ that was equaled or exceeded for indicated percentage of time } \\
\hline Percent & 90 & 95 & 99 \\
Discharge & 0.6 & 0.5 & 0.3 \\
\hline
\end{tabular}


Table 2. Summary of drainage-basin characteristics, and low-flow and flow-duration estimates for partial-record stations within the municipio of Caguas, Puerto Rico-Continued

\section{RÍO GRANDE DE LOÍZA BASIN-Continued \\ 50055150 Río Cañaboncito at Barrio Cañaboncito, Puerto Rico}

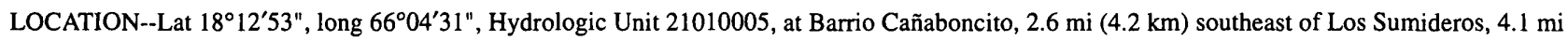
$(6.6 \mathrm{~km})$ northeast of Cerro Las Piñas, and $3.0 \mathrm{mi}(4.8 \mathrm{~km})$ southwest from Caguas plaza.

DRAINAGE AREA-- $1.6 \mathrm{mi}^{2}\left(4.2 \mathrm{~km}^{2}\right)$.

LOW-FLOW AND FLOW-DURATION ESTIMATES--Based on correlation of 8 base-flow measurements with concurrent base flows at gaging station 50055100 .

REMARKS--None.

LOW-FLOW CHARACTERISTICS

\begin{tabular}{cc}
\hline Low-flow characteristics & Discharge $\left(\mathrm{ft}^{3} / \mathrm{s}\right)$ \\
\hline 7-day, 2-year & 0.3 \\
7-day, 10-year & 0.1 \\
\hline
\end{tabular}

FLOW-DURATION CHARACTERISTICS

\begin{tabular}{lccc}
\hline \multicolumn{4}{c}{ Discharge $\left(\mathrm{ft}^{3} / \mathrm{s}\right)$ that was equaled or exceeded for indicated percentage of time } \\
\hline Percent & 90 & 95 & 99 \\
Discharge & 0.3 & 0.3 & 0.2 \\
\hline
\end{tabular}

50055330 Río Bairoa at Barrio Bairoa near Caguas, Puerto Rico

LOCATION-- $18^{\circ} 15^{\prime} 44^{\prime \prime}$, long $66^{\circ} 04^{\prime} 31^{\prime \prime}$, Hydrologic Unit 21010005 , at Barrio Bairoa, $0.5 \mathrm{mi}(0.8 \mathrm{~km})$ northwest of Las Carolinas, $2.8 \mathrm{mi}(4.5 \mathrm{~km})$ southwest of Cerro Marquesa, and $1.9 \mathrm{mi}(3.0 \mathrm{~km})$ northeast of Aguas Buenas plaza.

DRAINAGE AREA-- $3.0 \mathrm{mi}^{2}\left(7.8 \mathrm{~km}^{2}\right)$.

LOW-FLOW AND FLOW-DURATION ESTIMATES--Based on correlation of 8 base-flow measurements with concurrent base flows at gaging station 50053025 .

REMARKS--A diversion of $0.07 \mathrm{ft}^{3} / \mathrm{s}$ is made upstream of station for public water supply.

LOW-FLOW CHARACTERISTICS

\begin{tabular}{|c|c|c|c|}
\hline \multicolumn{2}{|c|}{ Low-flow characteristics } & \multicolumn{2}{|c|}{ Discharge $\left(\mathrm{ft}^{3} / \mathrm{s}\right)$} \\
\hline \multicolumn{2}{|c|}{ 7-day, 2-year } & \multicolumn{2}{|c|}{1.2} \\
\hline \multicolumn{2}{|c|}{ 7-day, 10-year } & \multicolumn{2}{|c|}{0.8} \\
\hline \multicolumn{4}{|c|}{ FLOW-DURATION CHARACTERISTICS } \\
\hline \multicolumn{4}{|c|}{ Discharge $\left(\mathrm{ft}^{3} / \mathrm{s}\right)$ that was equaled or exceeded for indicated percentage of time } \\
\hline Percent & 90 & 95 & 99 \\
\hline Discharge & 1.3 & 1.1 & 0.8 \\
\hline
\end{tabular}


Table 2. Summary of drainage-basin characteristics, and low-flow and flow-duration estimates for partial-record stations within the municipio of Caguas, Puerto Rico-Continued

\section{RÍO GRANDE DE LOIZZA BASIN-Continued}

\section{Río Cañas at Barrio Jagüeyes near Aguas Buenas, Puerto Rico}

LOCATION--Lat $18^{\circ} 16^{\prime} 54^{\prime \prime}$, long $66^{\circ} 04^{\prime} 41^{\prime \prime}$, Hydrologic Unit 21010005, at Barrio Jagüeyes, $2.8 \mathrm{mi}(4.5 \mathrm{~km})$ east of Cerro Marquesa, $1.8 \mathrm{mi}(2.9 \mathrm{~km})$ northwest of Las Carolinas, and $2.3 \mathrm{mi}(3.7 \mathrm{~km})$ northeast of Aguas Buenas plaza.

DRAINAGE AREA-- $1.4 \mathrm{mi}^{2}\left(3.6 \mathrm{~km}^{2}\right)$.

LOW-FLOW AND FLOW-DURATION ESTIMATES--Based on correlation of 8 base-flow measurements with concurrent base flows at gaging station 50058350

REMARKS--None.

LOW-FLOW CHARACTERISTICS

\begin{tabular}{cc}
\hline Low-flow characteristics & Discharge $\left(\mathrm{ft}^{3} / \mathrm{s}\right)$ \\
\hline 7-day, 2-year & 0.2 \\
7-day, 10-year & 0.1 \\
\hline
\end{tabular}

FLOW-DURATION CHARACTERISTICS

\begin{tabular}{lccc}
\hline \multicolumn{4}{c}{ Discharge $\left(\mathrm{ft}^{3} / \mathrm{s}\right)$ that was equaled or exceeded for indicated percentage of time } \\
\hline Percent & 90 & 95 & 99 \\
Discharge & 0.2 & 0.2 & 0.1 \\
\hline
\end{tabular}

50057750 Tributario de Río Cañas at Barrio Río Cañas, Puerto Rico

LOCATION--Lat $18^{\circ} 17^{\prime} 56^{\prime \prime}$, long $66^{\circ} 03^{\prime} 49^{\prime \prime}$, Hydrologic Unit 21010005, at Barrio Río Cañas, $3.3 \mathrm{mi}(5.3 \mathrm{~km})$ east of Cerro Marquesa, $1.7 \mathrm{mi}(2.7 \mathrm{~km})$ north of Las Carolinas, and $3.1 \mathrm{mi}(5.0 \mathrm{~km})$ northeast from Aguas Buenas plaza.

DRAINAGE AREA-- $0.7 \mathrm{mi}^{2}\left(1.8 \mathrm{~km}^{2}\right)$.

LOW-FLOW AND FLOW-DURATION ESTIMATES--Based on correlation of 8 base-flow measurements with concurrent base flows at gaging station 50058350 .

REMARKS--None.

LOW-FLOW CHARACTERISTICS

\begin{tabular}{cc}
\hline Low-flow characteristics & Discharge $\left(\mathrm{ft}^{3} / \mathrm{s}\right)$ \\
\hline 7-day, 2-year & 0.1 \\
7-day, 10-year & $<0.1$ \\
\hline
\end{tabular}

FLOW-DURATION CHARACTERISTICS

\begin{tabular}{lccc}
\multicolumn{4}{c}{ Discharge $\left(\mathrm{ft}^{3} / \mathrm{s}\right)$ that was equaled or exceeded for indicated percentage of time } \\
\hline Percent & 90 & 95 & 99 \\
Discharge & 0.1 & 0.1 & $<0.1$ \\
\hline
\end{tabular}


Table 2. Summary of drainage-basin characteristics, and low-flow and flow-duration estimates for partial-record stations within the municipio of Caguas, Puerto Rico-Continued

RÍO GRANDE DE LOIZZA BASIN-Continued

\section{Río Cañas at Barrio Jagüeyes near Río Cañas, Puerto Rico}

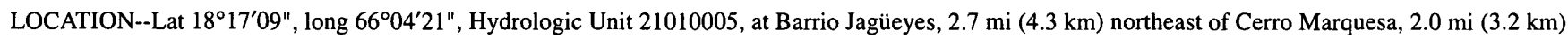
northwest of Las Carolinas, and $2.8 \mathrm{mi}(4.5 \mathrm{~km})$ northeast from Aguas Buenas plaza.

DRAINAGE AREA-- $2.7 \mathrm{mi}^{2}\left(7.1 \mathrm{~km}^{2}\right)$.

LOW-FLOW AND FLOW-DURATION ESTIMATES--Based on correlation of 9 base-flow measurements with concurrent base flows at gaging station 50058350

REMARKS--None.

LOW-FLOW CHARACTERISTICS

\begin{tabular}{cc}
\hline Low-flow characteristics & Discharge $\left(\mathrm{ft}^{3} / \mathrm{s}\right)$ \\
\hline 7-day, 2-year & 0.5 \\
7-day, 10-year & 0.2 \\
\hline
\end{tabular}

FLOW-DURATION CHARACTERISTICS

\begin{tabular}{lccc}
\hline \multicolumn{4}{c}{ Discharge $\left(\mathrm{ft}^{3} / \mathrm{s}\right)$ that was equaled or exceeded for indicated percentage of time } \\
\hline Percent & 90 & 95 & 99 \\
Discharge & 0.5 & 0.4 & 0.2 \\
\hline
\end{tabular}

50058010 Río Cañas at Highway 1 at Barrio Río Cañas, Puerto Rico

LOCATION--Lat $18^{\circ} 17^{\prime} 41^{\prime \prime}$, long $66^{\circ} 03^{\prime} 02^{\prime \prime}$, Hydrologic Unit 21010005, at Barrio Río Cañas at Highway 1, 2.7 mi (4.3 km) northeast of Las Carolinas, $4.2 \mathrm{mi}(6.8 \mathrm{~km})$ northeast of Cerro Marquesa, and $4.3 \mathrm{mi}(6.9 \mathrm{~km})$ northeast from Aguas Buenas plaza.

DRAINAGE AREA-- $3.6 \mathrm{mi}^{2}\left(9.3 \mathrm{~km}^{2}\right)$.

LOW-FLOW AND FLOW-DURATION ESTIMATES--Based on correlation of 8 base-flow measurements with concurrent base flows at gaging station 50058350 .

REMARKS--None.

LOW-FLOW CHARACTERISTICS

\begin{tabular}{cc}
\hline Low-flow characteristics & Discharge $\left(\mathrm{ft}^{3} / \mathrm{s}\right)$ \\
\hline 7-day, 2-year & 0.7 \\
7-day, 10-year & 0.3 \\
\hline
\end{tabular}

FLOW-DURATION CHARACTERISTICS

\begin{tabular}{lccc}
\hline \multicolumn{4}{c}{ Discharge $\left(\mathrm{ft}^{3} / \mathrm{s}\right)$ that was equaled or exceeded for indicated percentage of time } \\
\hline Percent & 90 & 95 & 99 \\
Discharge & 0.7 & 0.5 & 0.3 \\
\hline
\end{tabular}


Table 3. Principal features of public water-supply filtration plants, surface-water intakes, public waste-water treatment facilities, and effluent-discharge points

[Mgal/d, million gallons per day; est., estimate; --, no data]

\begin{tabular}{|c|c|c|c|c|c|c|}
\hline $\begin{array}{l}\text { Public water-supply } \\
\text { filtration plants and } \\
\text { surface-water intakes }\end{array}$ & Latitude $^{1}$ & Longitude $^{1}$ & $\begin{array}{l}\text { Safe yield } \\
\text { (Mgal/d) }\end{array}$ & $\begin{array}{l}\text { Water } \\
\text { available } \\
\text { for use }{ }^{3} \\
\text { (Mgal/d) }\end{array}$ & $\begin{array}{c}\text { Mean daily } \\
\text { withdrawal rate } \\
(\mathrm{Mgal} / \mathrm{d})\end{array}$ & Source stream \\
\hline $\begin{array}{l}\text { Quebrada de las Quebradillas } \\
\text { filtration plant (Caguas } \\
\text { filtration plant) }\end{array}$ & $18^{\circ} 11^{\prime} 38^{\prime \prime}$ & $66^{\circ} 03^{\prime} 44^{\prime \prime}$ & -- & -- & 6.5 & -- \\
\hline $\begin{array}{l}\text { Quebrada de las Quebradillas } \\
\text { pump station }\end{array}$ & $18^{\circ} 11^{\prime} 21^{\prime \prime}$ & $66^{\circ} 03^{\prime} 03^{\prime \prime}$ & 1.67 & 3.04 & -- & Río Turabo ${ }^{4}$ \\
\hline $\begin{array}{l}\text { Quebrada de las Quebradillas } \\
\text { gravity intake pump }\end{array}$ & $18^{\circ} 11^{\prime} 39^{\prime \prime}$ & $66^{\circ} 04^{\prime} 06^{\prime \prime}$ & 0.35 & 0.78 & -- & $\begin{array}{l}\text { Quebrada de las } \\
\text { Quebradillas }\end{array}$ \\
\hline Caguas Norte filtration plant & $18^{\circ} 14^{\prime} 52^{\prime \prime}$ & $66^{\circ} 01^{\prime} 19^{\prime \prime}$ & -- & -- & 5.8 & -- \\
\hline Caguas Norte pump station & $18^{\circ} 14^{\prime} 50^{\prime \prime}$ & $66^{\circ} 00^{\prime} 55^{\prime \prime}$ & -- & 14.0 & -- & $\begin{array}{l}\text { Río Grande de } \\
\text { Loíza }\end{array}$ \\
\hline San Salvador filtration plant & $18^{\circ} 08^{\prime} 16^{\prime \prime}$ & $66^{\circ} 02^{\prime} 10^{\prime \prime}$ & -- & -- & 0.3 & -- \\
\hline $\begin{array}{l}\text { San Salvador gravity intake } \\
\text { pump }\end{array}$ & $18^{\circ} 07^{\prime} 45^{\prime \prime}$ & $66^{\circ} 02^{\prime} 16^{\prime \prime}$ & 0.2 & -- & - & $\begin{array}{l}\text { Quebrada } \\
\text { Morena }\end{array}$ \\
\hline San Antonio filtration plant & $18^{\circ} 18^{\prime} 14^{\prime \prime}$ & $66^{\circ} 01^{\prime} 56^{\prime \prime}$ & -- & -- & ${ }^{5} 1.5$ & Lago Loíza \\
\hline $\begin{array}{l}\text { Public waste-water } \\
\text { treatment facilities and } \\
\text { effluent-discharge points }\end{array}$ & Latitude & Longitude & $\begin{array}{l}\text { Discharge } \\
\text { capacity } \\
\text { (Mgal/d) }\end{array}$ & $\begin{array}{l}\text { Meandaily } \\
\text { discharge } \\
\text { rate (est.1998) } \\
\text { (Mgal/d) }\end{array}$ & Receiving stream & \\
\hline $\begin{array}{l}\text { Caguas regional waste-water } \\
\text { treatment plant }\end{array}$ & $18^{\circ} 15^{\prime} 49^{\prime \prime}$ & $66^{\circ} 01^{\prime} 39^{\prime \prime}$ & 12.0 & -- & -- & \\
\hline $\begin{array}{l}\text { Caguas regional waste-water } \\
\text { treatment plant outlet }\end{array}$ & $18^{\circ} 15^{\prime} 40^{\prime \prime}$ & $66^{\circ} 01^{\prime} 27^{\prime \prime}$ & -- & 7.6 & Río Bairoa & \\
\hline $\begin{array}{l}\text { Borinquen waste-water } \\
\text { treatment plant }\end{array}$ & $18^{\circ} 10^{\prime} 59^{\prime \prime}$ & $66^{\circ} 02^{\prime} 56^{\prime \prime}$ & 0.30 & -- & -- & \\
\hline $\begin{array}{l}\text { Borinquen waste-water } \\
\text { treatment plant outlet }\end{array}$ & $18^{\circ} 10^{\prime} 59^{\prime \prime}$ & $66^{\circ} 02^{\prime} 53^{\prime \prime}$ & -- & 0.04 & Río Turabo & \\
\hline $\begin{array}{l}\text { Las Carolinas waste-water } \\
\text { treatment plant }\end{array}$ & $18^{\circ} 15^{\prime} 10^{\prime \prime}$ & $66^{\circ} 04^{\prime} 17^{\prime \prime}$ & 0.22 & -- & -- & \\
\hline $\begin{array}{l}\text { Las Carolinas waste-water } \\
\text { treatment plant outlet }\end{array}$ & $18^{\circ} 15^{\prime} 05^{\prime \prime}$ & $66^{\circ} 04^{\prime} 16^{\prime \prime}$ & -- & 0.04 & Río Cagüitas & \\
\hline
\end{tabular}

1 Datum for latitude and longitude is NAD 27.

${ }^{2}$ Safe-yield values from Black and Veatch (1996).

${ }^{3}$ Water available for use is estimated using the 0-99 flow-duration estimate derived in the present study.

${ }^{4}$ During this study, Río Turabo was being channelized. When completed, the left stream bank levee may isolate the pump station intake from the Rio Turabo, limiting water supply to the intake to discharge from Quebrada Beatriz and an unnamed tributary draining the Las Praderas sector, which have an estimated Q-99 flow duration of $0.32 \mathrm{Mgal} / \mathrm{d}$.

${ }^{5}$ Plant was under construction during 1997-98. Rate stated corresponds to September 2000; during 1998, rate may have been less than stated. 
18 Surface-Water, Water-Quality, and Ground-Water Assessment of the Municipio of Caguas, Puerto Rico, 1997-99 


\title{
CHAPTER B:
}

\section{Sanitary Quality of Surface Water During Base-Flow Conditions in the Municipio of Caguas, Puerto Rico, 1998-99}

\author{
By Fernando Gómez-Gómez, Mario L. Oliveras-Feliciano, and Carlos Conde-Costas
}

\section{BACKGROUND}

Water-quality standards for surface waters in Puerto Rico have been established by the Puerto Rico Environmental Quality Board (Junta de Calidad Ambiental de Puerto Rico, 1990) on the basis of the designated use (for example, fishing, source of raw water for public supply, and secondary contact recreation, among others). All perennial fresh surface waters in Puerto Rico inland of their estuary segments have been classified as Class SD waters. This classification includes surface water intended for use (or with the potential for use) as a raw source of public water supply, for propagation and preservation of desirable aquatic species, and for primary and secondary contact recreation. The sanitary quality standard for Class SD surface water is based on the fecal coliform or total coliform indicator bacteria (Junta de Calidad Ambiental de Puerto Rico, 1990).

With the exception of surface waters to be used for primary contact recreation, the sanitary quality standard of Class SD-designated use surface waters is based on total coliform and fecal coliform bacteria concentrations as follows: the geometric mean concentration of at least five samples obtained in sequential order should not exceed 10,000 colonies per 100 milliliters $(\mathrm{mL})$ for total coliform bacteria, or 2,000 colonies per $100 \mathrm{~mL}$ for fecal coliform bacteria, and not more than 20 percent of the samples (one in a set of five) should exceed 4,000 colonies per $100 \mathrm{~mL}$ of fecal coliform bacteria (Junta de Calidad Ambiental de Puerto Rico, 1990, Article 3, Section 2.4, as amended July 20,1990). Unlike other regions in the United States, the Puerto Rico regulations do not constrain the time period during which the sequential samples must be obtained. In Puerto Rico, however, these standards are applicable only to samples taken when streamflows are greater than the 7-day, 2-year (7-d, Q-2y) discharge (Junta de Calidad Ambiental de Puerto Rico, 1990). The 7-d, Q-2y discharge corresponds to the discharge at the 2-year recurrence interval taken from a frequency curve of annual values of the lowest mean discharge for 7 consecutive days (the 7-day low flow).

Typical concentrations of the two most common indicator bacteria in contaminated water are given in table 4. Fecal coliform and fecal streptococcus bacteria are not pathogenic, but have been correlated to the presence of several waterborne, infectious disease-causing organisms present in wastes from warm-blooded animals (including humans). Thus, the concentration of these indicator bacteria is a measure of water safety for consumption or for body contact.

Contamination sources that affect stream sanitary quality during base-flow conditions are distinct for urbanized and rural areas of Caguas. In urbanized areas, probable major sources of fecal contamination are illegal discharge of sewage to storm-water drains, especially within the older sectors of the city of Caguas, overflows from sewer mains into the storm-water drains as a result of malfunctioning sanitary sewer ejectors or clogged mains, ruptured sewer mains, and seepage from sewer mains into the local aquifer. In rural areas, major sources of fecal contamination include gray-water discharges (gray water includes waste water except sanitary wastes) from residential and commercial establishments along stream channels, septic tank seepage or overflows, feces contamination directly into streams from unfenced livestock, and runoff from restrained livestock pens near stream courses. Another potential 
Table 4. Ranges of fecal indicator bacteria concentrations typically found in contaminated surface water (modified from Myers and Sylvester (1997))

\begin{tabular}{ll}
\hline Bacteriological group & $\begin{array}{c}\text { Fecal-contaminated surface water, } \\
\text { colonies per } 100 \text { milliliters }\end{array}$ \\
\hline Fecal coliform & 200 to greater than 2 million \\
Fecal streptococcus & 400 to greater than 1 million \\
\hline
\end{tabular}

source of fecal contamination is runoff and groundwater seepage from the municipal landfill at Barrio Turabo (Gómez-Gómez, 1980) (plate 1).

Baseline data on the sanitary quality of surface waters within the municipio of Caguas are primarily from the long-term monitoring stations at the Río Grande de Loíza, the Río Cagüitas, and the Río Bairoa (USGS stations 50055000, 50055250, and 50055400, respectively, in plate 1), and the reservoir sampling station at Lago Loíza located approximately 0.5 mile downstream of the confluence of the Río Gurabo (USGS station 50057500 in plate 1). Systematic sampling for selected physical, chemical, and bacteriological parameters has been done at these stations by the USGS in cooperation with the Puerto Rico Environmental Quality Board since 1978 (data available in USGS annual Water Data Report series). The trend of geometric mean concentrations for fecal coliform bacteria of five sequential samples at these stations for the period 1984 to 1998 is shown in figures $2 \mathrm{a}, 2 \mathrm{~b}$, and $2 \mathrm{c}$ (refer to plate 1 for locations).

Using this historical database to initially assess the sanitary quality of streams in the Caguas area, it was determined that (1) there has been no substantial improvement towards achieving the Puerto Rico sanitary water-quality goals established for fresh surface waters, since the regulations were enacted (Junta de Calidad Ambiental de Puerto Rico, 1990);

(2) downstream of the city of Caguas the streams are highly contaminated; and (3) these baseline fecal coliform data are insufficient to identify sources of fecal contamination within the study area. The data also indicate that the geometric mean concentration of fecal coliform indicator bacteria for five sequential samples consistently range from 2,000 to 30,000 colonies per $100 \mathrm{~mL}$ at the Río Grande de Loíza sampling station and 10,000 to 100,000 colonies per $100 \mathrm{~mL}$ at the Río Caguiitas and the Río Bairoa sampling stations. The latter two sampling stations have geometric mean fecal coliform concentrations substantially above the sanitary quality goal of 2,000 colonies per $100 \mathrm{~mL}$ established by Junta de Calidad Ambiental de Puerto Rico (1990).

At the Lago Loíza reservoir, fecal coliform samples were obtained on a less frequent basis (three times per year in/or about the months of March, July, and December) since 1978. During these months, there is typically less rainfall than in the preceding months. Thus, samples obtained at the reservoir correspond primarily to base-flow conditions. The fecal coliform geometric mean concentrations at the sampling station in the Lago Loíza reservoir have, for the most part, been below 2,000 colonies per $100 \mathrm{~mL}$ since 1990, with the exception of a 2 -year period from May 1994 to July 1996 (fig. 2d). The geometric mean values plotted in figure $2 \mathrm{~d}$ were calculated from analytical results of five samples (each point on the curve corresponding to the geometric mean of the four previous sampled dates plus the one plotted). The period shown in figure $2 \mathrm{~d}$ includes samples obtained from March, July, and November 1993; March, May, and November 1994; March, July, and November 1995; and March and July 1996. Streamflows were substantially below average for most months from August 1993 through November 1994 and thus, it is likely that the high concentration of fecal coliform bacteria at the reservoir sampling site was caused by several conditions that existed during this dry period. Among these conditions were low dilution of fecalcontaminated waste discharges in streams and wastewater discharges to the sanitary sewers during a waterrationing period in 1994 that were characterized by high loads over short periods and caused overflows in sewer mains and below-average retention times at waste-water treatment facilities. In addition, suspended sediment concentrations at streams, which 
serve indirectly as flocculating agents, were significantly below that of average rainfall/runoff years from 1994 to 1996 (Díaz and others, 1995, 1996, and 1997).

\section{PURPOSE AND SCOPE}

A survey of stream sanitary quality was conducted by the USGS in cooperation with the Municipio Autónomo de Caguas to define the extent of fecal contamination in streams. The assessment was made by obtaining and analyzing samples for fecal coliform and fecal streptococcus bacteria from 38 stream locations during base-flow conditions between May 1998 and July 1999. Although the synoptic surveys were conducted in streams that have headwaters outside the municipal boundary, most of the sampling was conducted within the territorial limits of the municipio of Caguas. The study area included $73.6 \mathrm{mi}^{2}$ of drainage area, of which $58.9 \mathrm{mi}^{2}$ are within the municipio of Caguas.

This chapter describes the methods and techniques used in conducting the survey at the 38 sampling stations, and provides interpretations of the fecal coliform and fecal streptococcus indicator bacteria concentrations by developing a classification procedure to rank the sanitary quality of stream courses. The data were incorporated into a thematic map (plate 1) that also includes other important hydrologic features to (a) serve as an initial source of information to guide future efforts by municipal and Commonwealth authorities in implementing measures to enhance the sanitary quality of contaminated streams and conserve those with an acceptable quality; (b) provide reliable scientific information to planners and managers of the water and biological resources; and (c) encourage local citizens to serve as stewards of water resources within their municipality.

\section{METHODOLOGY}

\section{Field-Data Collection}

Water samples for fecal coliform and fecal streptococcus analyses were obtained from a total of 40 locations at streams with drainage to or within the municipio of Caguas and one sampling station within the Lago Loíza reservoir. Sampling was conducted at all stream stations using the "hand-dip" method (Britton and Greeson, 1989). This method is applicable to the stream low-flow conditions used in this study. This sampling method consists of dipping a sterile narrow-mouth borosilicate $99-\mathrm{mL}$ bottle 1 to 2 inches below the water surface with the bottle opening pointed slightly upward towards the current and with the hand and arm on the downstream side of the bottle. Sampling at the Lago Loíza reservoir station was conducted using the hand-dip method at the center of the cross section.

Most samples were obtained during stream base-flow periods between May 1998 and July 1999 for two hydrologic conditions (1) near the annual stream low-flow discharge, and (2) during base-flow conditions after a rainfall event. Samples obtained during these flow regimes were used in developing a classification methodology to rank approximately 137 miles (mi) of stream courses within the municipio of Caguas or with drainage into Caguas. During stream base-flow conditions, fecal contamination in streams is principally from sources that discharge directly to stream channels (especially during the period of annual low flow), or are washed into stream channels from sources adjacent (hundreds of feet) to stream banks. To conduct the assessment, 40 stream sampling stations were established. Only 34 stations were sampled on both occasions at base flow, 3 were sampled twice, but only 1 sample complied with the requisite of base-flow conditions during the sampling round, 2 were sampled only once during rising stage, and 1 , only once, when flow was stagnant. As a result, classification of the sanitary condition of stream courses was made using data obtained from the 37 stream sampling stations, where at least one sample was obtained at base-flow conditions. In addition, two samples were obtained from Lago Loíza, on two different occasions, for a total of 38 surface-water sampling stations for this study.

Instantaneous discharge measurements were made simultaneously during sample collection using the current meter method (Carter and Davidian, 1968) except in a few cases where flow was stagnant or streamflow discharge was on a rapid rising stage from localized rainfall. Sixteen of the sampling stations 
correspond with stations also used in the surface-water low-flow statistics assessment (Chapter A). Among the low-flow statistics estimated at these sampling stations is the 7-day, 2-year discharge. This statistical discharge value is included with the instantaneous discharge measurements made during the sample runs to indicate the minimum discharge rate at which the fecal coliform concentration standards are applicable in Puerto Rico (Junta de Calidad Ambiental de Puerto Rico, 1990).

\section{Analytical Techniques}

The analytical procedure used to measure fecal coliform and fecal streptococcus bacteria concentrations consisted of the membrane-filter method immediate incubation test following standard USGS procedures (Britton and Greeson, 1989; Myers and Sylvester, 1997). Sterile buffered water, culture media, and other culture specific reagents (for example, rosolic acid crystals, $0.2 \mathrm{~N}$ sodium hydroxide for fecal coliform tests, and triphenyltetrazolium crystals for fecal streptococcus tests) were provided by the USGS Water Quality and Research Laboratory in Ocala, Florida. Hydrated incubation media in petri dishes and sterile sample bottles were prepared at the Caribbean District laboratory in Guaynabo, Puerto Rico.

Based on previous sampling experience, dilution ratios for membrane filtration analyses at each station were prepared to maximize the probability of obtaining about 20 to 60 colonies per filter for fecal coliform bacteria and 20 to 100 colonies per filter for fecal streptococcus bacteria. If colony counts were not in the ideal range, concentrations were reported as non-ideal. If the number of colonies per $100 \mathrm{~mL}$ from the highest dilution ratio of three sample volumes filtered at a station was above the ideal plate count, the greater than symbol $(>)$ preceding the number of colonies per $100 \mathrm{~mL}$ indicates that the concentration was estimated from a non-ideal high plate count. If the number of colonies per $100 \mathrm{~mL}$ from the lowest dilution ratio of three sample volumes filtered at a station was below the ideal plate count, the less than symbol (<) preceding the number of colonies per 100 $\mathrm{mL}$ indicates that the concentration was estimated from a non-ideal low plate count. With few exceptions, most samples were processed and incubated immediately after collection using the field laboratory. Where the field laboratory was not used, samples were preserved in an ice chest (ice water) at 1 to $4{ }^{\circ} \mathrm{C}$ and processed at the Caribbean District laboratory within 6 hours of sample collection.

The quality-assurance and quality-control (QA/QC) protocols for bacteriological analyses included (a) incubation of sterile buffered water in culture media at the Caribbean District laboratory as a check primarily on the sterile conditions of buffer, media, and filters; (b) field blanks to check sterile conditions of field equipment; and (c) processing of duplicate samples as a check on dilution procedures and variability of bacteriological concentrations resulting from dip sampling. Most QA/QC samples (on average 90 percent) consisted of laboratory or field blanks for parts (a) and (b). Duplicate sample dilutions were only two per sample run. Overall, the results for part (a) should be negative (no development of colonies; if positive, the media sample petri dishes and buffered-water dilution bottles are not acceptable for use). Results for (b) should be negative; if not, analytical results of samples obtained between negative QA/QC blanks (before and after the positive blank) are reviewed for suspect data results (for example, high counts or significant discrepancy between the number of colonies developed for sample dilutions with ideal and non-ideal counts). Anomalous data are not reported. For this study, no bacteriological results were discarded. Results are presented in table 5. Duplicate samples obtained as part of this survey were within the 15 percent of the original sample, which is typical for duplicate samples having fecal coliform and fecal streptococcus concentrations in the range of 100 to low thousand colonies per $100 \mathrm{~mL}$. Relative percent difference (RPD) of primary and duplicate samples are calculated as:

$$
\mathrm{RPD}=\mathrm{S} 1-\mathrm{S} 2 /[(\mathrm{S} 1+\mathrm{S} 2) / 2] \times 100,
$$

where $S 1$ is the primary sample and $S 2$ is the duplicate sample of colony counts in media plates with the number of colonies in the ideal range of 20 to 60 for fecal coliform and 20 to 100 for fecal streptococcus. 
Table 5. Fecal coliform and fecal streptococcus bacteria concentrations at selected sites in the municipio of Caguas, Puerto Rico

[*, indicates non-ideal plate count, either the number of colonies developed were less than ideal number (dilution too high) or greater than ideal number (dilution too low); ${ }^{\dagger}$, sample not used in station ranking, only two with lowest discharge; Pre-fair, presumed fair ranking; Pre-poor, presumed poor ranking; Pre-acceptable, presumed acceptable ranking; RS, stream in rapid rising stage not measured; >, greater than; <, less than; mL, milliliters; $\mathrm{ft}^{3} / \mathrm{s}$, cubic feet per second; $\mathrm{mi}^{2}$, square mile; Instant, instantaneous; 7-d, Q-2, 7-day, 2-year low flow; --, no data]

\begin{tabular}{|c|c|c|c|c|c|c|c|c|c|}
\hline $\begin{array}{l}\text { Sample site } \\
\text { USGS } \\
\text { identification } \\
\text { number }\end{array}$ & Site name & $\begin{array}{l}\text { Sample } \\
\text { date } \\
(\mathrm{m} / \mathrm{d} / \mathrm{y})\end{array}$ & Time & $\begin{array}{l}\text { Fecal } \\
\text { coliform } \\
\text { colonies } \\
\text { per } \\
100 \mathrm{~mL}\end{array}$ & $\begin{array}{l}\text { Fecal } \\
\text { streptococcus } \\
\text { colonies per } \\
100 \mathrm{~mL}\end{array}$ & $\begin{array}{l}\text { Instant } \\
\text { dis- } \\
\text { charge } \\
\left(\mathrm{ft}^{3} / \mathrm{s}\right)\end{array}$ & $\begin{array}{c}7-\mathrm{d}, 0-2 \\
\text { mean } \\
\text { daily } \\
\text { dis- } \\
\text { charge } \\
\left(\mathrm{ft}^{3} / \mathrm{s}\right)\end{array}$ & $\begin{array}{c}\text { Drainage } \\
\text { area } \\
\left(\mathrm{mi}^{2}\right)\end{array}$ & $\begin{array}{l}\text { Station } \\
\text { sanitary } \\
\text { quality } \\
\text { ranking }\end{array}$ \\
\hline \multirow[t]{2}{*}{50052795} & $\begin{array}{l}\text { Quebrada Janer at Barrio } \\
\text { Tomás de Castro }\end{array}$ & $6 / 3 / 98$ & 1230 & 4,000 & 150 & 0.02 & -- & 0.43 & Fair \\
\hline & & $11 / 24 / 98$ & 1400 & 220 & 155 & 0.02 & -- & -- & -- \\
\hline \multirow[t]{2}{*}{50052850} & $\begin{array}{l}\text { Quebrada Las Bambúas at } \\
\text { Barrio Tomás de Castro }\end{array}$ & $5 / 27 / 98$ & 1300 & 22,000 & 41,000 & 1.1 & -- & 1.36 & Poor \\
\hline & & $11 / 24 / 98$ & 1300 & 2,700 & 480 & 0.9 & - & -- & -- \\
\hline \multirow[t]{2}{*}{50052860} & $\begin{array}{l}\text { Quebrada Las Bambúas } \\
\text { near Caguas }\end{array}$ & $5 / 27 / 98$ & 1200 & 36,000 & 42,000 & 2.2 & -. & 1.78 & Poor \\
\hline & & $11 / 24 / 98$ & 1030 & 16,000 & 750 & 1.2 & -- & -- & -- \\
\hline \multirow[t]{2}{*}{50052925} & $\begin{array}{l}\text { Río Turabo at Barrio San } \\
\text { Salvador }\end{array}$ & $5 / 13 / 98$ & 1230 & 120 & 120 & 3.2 & 1.6 & 1.40 & Fair \\
\hline & & $9 / 17 / 98$ & 1330 & 2,700 & 530 & 8.4 & -- & -- & -- \\
\hline \multirow[t]{2}{*}{50052935} & $\begin{array}{l}\text { Quebrada Morena at Barrio } \\
\text { San Salvador }\end{array}$ & $5 / 13 / 98$ & 1330 & 560 & 140 & 2.9 & -- & 0.93 & Acceptable \\
\hline & & $9 / 17 / 98$ & 1200 & 500 & 200 & 3.3 & -- & $\cdots$ & -- \\
\hline \multirow[t]{2}{*}{50052950} & $\begin{array}{l}\text { Quebrada Maracai at } \\
\text { Barrio San Salvador }\end{array}$ & $5 / 13 / 98$ & 1130 & 300 & 300 & 5.1 & 2.6 & 2.33 & Acceptable \\
\hline & & $9 / 17 / 98$ & 1400 & 280 & 210 & 5.5 & -- & -- & -- \\
\hline \multirow[t]{2}{*}{50052975} & $\begin{array}{l}\text { Río Turabo near Barrio } \\
\text { San Salvador }\end{array}$ & $5 / 13 / 98$ & 1430 & 470 & 140 & 9.8 & -- & 5.50 & Acceptable \\
\hline & & $9 / 17 / 98$ & 1230 & $560^{*}$ & 220 & 18 & -- & -- & -- \\
\hline \multirow[t]{2}{*}{50053025} & $\begin{array}{l}\text { Río Turabo above } \\
\text { Borinquen }\end{array}$ & $5 / 13 / 98$ & 1000 & $910^{*}$ & 280 & 12 & 5.5 & 7.17 & Acceptable \\
\hline & & $9 / 17 / 98$ & 1430 & 440 & 190 & 23 & -- & -- & -- \\
\hline \multirow[t]{2}{*}{50053060} & $\begin{array}{l}\text { Río Turabo at Highway } \\
765 \text {, Barrio Borinquen }\end{array}$ & $5 / 12 / 98$ & 1340 & 420 & 360 & 14 & 5.6 & 8.10 & Acceptable \\
\hline & & $9 / 17 / 98$ & 1300 & 590 & 250 & 15 & -- & -- & -- \\
\hline \multirow[t]{2}{*}{50053080} & $\begin{array}{l}\text { Quebrada Naranjito at } \\
\text { Barrio Borinquen }\end{array}$ & $5 / 12 / 98$ & 1240 & 5,300 & 440 & 0.9 & $<0.1$ & 2.25 & Poor \\
\hline & & $9 / 17 / 98$ & 1300 & 5,400 & 450 & 1.1 & -- & -- & -- \\
\hline \multirow[t]{2}{*}{50053085} & $\begin{array}{l}\text { Tributary at Barrio } \\
\text { Borinquen to Río Turabo } \\
\text { at Highway } 765\end{array}$ & $5 / 12 / 98$ & 1140 & 4,500 & 450 & 0.1 & -- & 0.39 & Poor \\
\hline & & $9 / 17 / 98$ & 1600 & 2,300 & 340 & 0.2 & -- & -- & -- \\
\hline \multirow[t]{3}{*}{50053150} & $\begin{array}{l}\text { Quebrada Sonadora at } \\
\text { Barrio Beatriz }\end{array}$ & $5 / 14 / 98$ & 1200 & 420 & 180 & 1.0 & 0.3 & 1.20 & Acceptable \\
\hline & & $9 / 17 / 98$ & 1500 & 3,700 & 580 & $2.0^{\dagger}$ & -- & -- & - \\
\hline & & $7 / 6 / 99$ & 1110 & 250 & 190 & 1.3 & -- & -- & -- \\
\hline
\end{tabular}


Table 5. Fecal coliform and fecal streptococcus bacteria concentrations at selected sites in the municipio of Caguas, Puerto Rico-Continued

\begin{tabular}{|c|c|c|c|c|c|c|c|c|c|}
\hline $\begin{array}{l}\text { Sample site } \\
\text { USGS } \\
\text { identification } \\
\text { number }\end{array}$ & Site name & $\begin{array}{l}\text { Sample } \\
\text { date } \\
(\mathrm{m} / \mathrm{d} / \mathrm{y})\end{array}$ & Time & $\begin{array}{c}\text { Fecal } \\
\text { coliform } \\
\text { colonies } \\
\text { per } \\
100 \mathrm{~mL}\end{array}$ & $\begin{array}{l}\text { Fecal } \\
\text { streptococcus } \\
\text { colonies per } \\
100 \mathrm{~mL}\end{array}$ & $\begin{array}{l}\text { Instant } \\
\text { dis- } \\
\text { charge } \\
\left(\mathrm{ft}^{3} / \mathrm{s}\right)\end{array}$ & $\begin{array}{c}7-\mathrm{d}, 0-2 \\
\text { mean } \\
\text { daily } \\
\text { dis- } \\
\text { charge } \\
\left(\mathrm{ft}^{3} / \mathrm{s}\right)\end{array}$ & $\begin{array}{l}\text { Drainage } \\
\text { area } \\
\left(\mathrm{mi}^{2}\right)\end{array}$ & $\begin{array}{l}\text { Station } \\
\text { sanitary } \\
\text { quality } \\
\text { ranking }\end{array}$ \\
\hline \multirow[t]{2}{*}{50053200} & $\begin{array}{l}\text { Quebrada Beatriz at Barrio } \\
\text { Beatriz }\end{array}$ & $6 / 3 / 98$ & 1030 & $110 *$ & $130 *$ & 1.9 & 0.9 & 4.42 & Good \\
\hline & & $7 / 6 / 99$ & 1225 & 130 & 280 & 3.4 & -- & -- & -- \\
\hline \multirow[t]{2}{*}{50053250} & $\begin{array}{l}\text { Tributary to Quebrada } \\
\text { Beatriz at Highway } 765\end{array}$ & $5 / 12 / 98$ & 1045 & 2,600 & 770 & 0.2 & -- & 0.46 & Poor \\
\hline & & $9 / 17 / 98$ & 1600 & 5,100 & 630 & 0.3 & -- & -- & -- \\
\hline \multirow[t]{2}{*}{50053925} & $\begin{array}{l}\text { Quebrada de las } \\
\text { Quebradillas at Divisoria }\end{array}$ & $6 / 4 / 98$ & 1200 & 440 & 220 & 1.3 & -. & 2.05 & Acceptable \\
\hline & & $3 / 3 / 99$ & 1040 & 200 & 240 & 3. & -- & -- & -- \\
\hline \multirow[t]{2}{*}{50053950} & $\begin{array}{l}\text { Quebrada de las } \\
\text { Quebradillas at Barrio } \\
\text { Beatriz }\end{array}$ & $6 / 4 / 98$ & 1100 & 500 & 220 & 3.0 & 1.0 & 3.88 & Acceptable \\
\hline & & $3 / 3 / 99$ & 0950 & 470 & 290 & 6.6 & -- & -- & -- \\
\hline \multirow[t]{3}{*}{50053975} & $\begin{array}{l}\text { Quebrada de las } \\
\text { Quebradillas above dam }\end{array}$ & $3 / 2 / 99$ & 1110 & 6,500 & 7,200 & 12 & 1.8 & -- & Fair \\
\hline & & $5 / 26 / 98$ & 1130 & $720 *$ & 890 & 9.2 & -- & 6.28 & -- \\
\hline & & $6 / 4 / 98$ & 1300 & 470 & $180^{*}$ & RS & -- & -- & -- \\
\hline \multirow[t]{3}{*}{50054050} & $\begin{array}{l}\text { Quebrada de las } \\
\text { Quebradillas near mouth }\end{array}$ & $5 / 26 / 98$ & 1020 & 2,600 & 860 & 13 & -- & 7.05 & Poor \\
\hline & & $9 / 17 / 98$ & 1030 & 2,400 & 750 & 6.2 & -- & -- & -- \\
\hline & & $3 / 2 / 99$ & 1225 & 4,200 & 1,000 & 7.1 & -- & -- & -- \\
\hline \multirow[t]{2}{*}{50054100} & $\begin{array}{l}\text { Río Turabo at Barrio } \\
\text { Turabo }\end{array}$ & $5 / 14 / 98$ & 1330 & 430 & $60 *$ & 15 & -- & 24.50 & Pre-fair \\
\hline & & $9 / 17 / 98$ & 1130 & 2,600 & 980 & RS & -- & -- & -- \\
\hline \multirow[t]{3}{*}{50054300} & $\begin{array}{l}\text { Río Turabo at Villa del Rey } \\
\text { near Caguas }\end{array}$ & $5 / 29 / 98$ & 1130 & 340 & 80 & RS & 3.4 & 25.08 & Fair \\
\hline & & $6 / 2 / 98$ & 1200 & $40 *$ & $<10^{*}$ & 11 & -- & -- & -- \\
\hline & & $9 / 17 / 98$ & 1100 & 30,000 & 11,000 & 37 & -- & -- & -- \\
\hline \multirow[t]{2}{*}{50054500} & Río Turabo at Caguas & $5 / 29 / 98$ & 1230 & 2,600 & 2,200 & RS & -- & 29.42 & Pre-poor \\
\hline & & $9 / 17 / 98$ & 0930 & 4,500 & 500 & 40 & -- & -- & -- \\
\hline \multirow[t]{2}{*}{50055000} & $\begin{array}{l}\text { Río Grande de Loíza at } \\
\text { Caguas }\end{array}$ & $5 / 11 / 98$ & 1400 & 600 & 45 & 78 & 32 & 90.3 & Fair \\
\hline & & $6 / 2 / 98$ & 0900 & 3,400 & $170 *$ & 70 & -- & -- & -- \\
\hline \multirow[t]{3}{*}{50055120} & $\begin{array}{l}\text { Río Cagüitas at Highway } \\
156 \text { near Caguas }\end{array}$ & $5 / 16 / 98$ & 1000 & 570 & 380 & 3.5 & 0.5 & 7.48 & Acceptable \\
\hline & & $9 / 18 / 98$ & 1100 & 430 & 320 & 1.1 & -- & -- & -- \\
\hline & & $7 / 15 / 99$ & 1030 & $2,100^{\dagger}$ & 4,200 & 10 & -- & $-\cdot$ & -- \\
\hline 50055092 & $\begin{array}{l}\text { Río Cagüitas at Highway } \\
777\end{array}$ & $7 / 15 / 99$ & 0900 & 5,800 & 11,000 & RS & -- & 2.81 & Not ranked \\
\hline 50055097 & $\begin{array}{l}\text { Quebrada Sanjelo at } \\
\text { Highway } 777\end{array}$ & $7 / 15 / 99$ & 0945 & $\begin{array}{r}130,000 \\
*\end{array}$ & $110,000 *$ & RS & -- & 1.41 & Not ranked \\
\hline \multirow[t]{2}{*}{50055130} & $\begin{array}{l}\text { Quebrada Algarrobo at } \\
\text { Barrio Cañabón }\end{array}$ & $5 / 16 / 98$ & 1130 & 450 & 470 & 0.1 & -- & 0.46 & Acceptable \\
\hline & & $9 / 18 / 98$ & 1600 & $70 *$ & 260 & 0.1 & -- & -- & -- \\
\hline
\end{tabular}


Table 5. Fecal coliform and fecal streptococcus bacteria concentrations at selected sites in the municipio of Caguas, Puerto Rico-Continued

\begin{tabular}{|c|c|c|c|c|c|c|c|c|c|}
\hline $\begin{array}{c}\text { Sample site } \\
\text { USGS } \\
\text { identification } \\
\text { number }\end{array}$ & Site name & $\begin{array}{c}\text { Sample } \\
\text { date } \\
(\mathrm{m} / \mathrm{d} / \mathrm{v})\end{array}$ & Time & $\begin{array}{c}\text { Fecal } \\
\text { coliform } \\
\text { colonies } \\
\text { per } \\
100 \mathrm{~mL}\end{array}$ & $\begin{array}{l}\text { Fecal } \\
\text { streptococcus } \\
\text { colonies per } \\
100 \mathrm{~mL}\end{array}$ & $\begin{array}{l}\text { Instant } \\
\text { dis- } \\
\text { charge } \\
\left(\mathrm{ft}^{3} / \mathrm{s}\right)\end{array}$ & $\begin{array}{c}\text { 7-d, } 0-2 \\
\text { mean } \\
\text { daily } \\
\text { dis- } \\
\text { charge } \\
\left(\mathrm{ft}^{3} / \mathrm{s}\right)\end{array}$ & $\begin{array}{c}\text { Drainage } \\
\text { area } \\
\left(\mathrm{mi}^{2}\right)\end{array}$ & $\begin{array}{l}\text { Station } \\
\text { sanitary } \\
\text { quality } \\
\text { ranking }\end{array}$ \\
\hline \multirow[t]{2}{*}{50055135} & $\begin{array}{l}\text { Quebrada Algarrobo at } \\
\text { mouth }\end{array}$ & $5 / 27 / 98$ & 1000 & 40 & 100 & $\begin{array}{l}\text { Stagnant } \\
\text { water }\end{array}$ & -- & 1.24 & $\begin{array}{c}\text { Pre- } \\
\text { acceptable }\end{array}$ \\
\hline & & $9 / 18 / 98$ & 1600 & 260 & 720 & 0.2 & -- & -- & -- \\
\hline \multirow[t]{2}{*}{50055150} & $\begin{array}{l}\text { Río Cañaboncito at Barrio } \\
\text { Cañaboncito }\end{array}$ & $5 / 21 / 98$ & 1030 & 3,500 & 660 & 0.5 & 0.3 & 1.63 & Poor \\
\hline & & $9 / 18 / 98$ & 1130 & 6,500 & 620 & 0.7 & -- & -- & - \\
\hline \multirow[t]{2}{*}{50055160} & $\begin{array}{l}\text { Río Cañaboncito near } \\
\text { mouth }\end{array}$ & $5 / 21 / 98$ & 1130 & 560 & 340 & 0.5 & -- & 3.14 & Acceptable \\
\hline & & $9 / 18 / 98$ & 1310 & 470 & $100 *$ & 1.0 & -- & -- & -- \\
\hline \multirow[t]{2}{*}{50055170} & Río Cagüitas near Caguas & $5 / 21 / 98$ & 1230 & 480 & $170 *$ & 2.7 & -- & 8.30 & Acceptable \\
\hline & & $9 / 18 / 98$ & 1230 & 610 & 130 & 1.5 & -- & - & -- \\
\hline \multirow[t]{2}{*}{50055250} & $\begin{array}{l}\text { Río Cagüitas at Highway } \\
30 \text { at Caguas }\end{array}$ & $6 / 2 / 98$ & 1000 & 30,000 & 2,800 & 5.1 & -- & 14.15 & Poor \\
\hline & & $9 / 18 / 98$ & 1430 & $\begin{array}{r}90,000 \\
*\end{array}$ & 9,300 & 13 & -- & -- & -- \\
\hline \multirow[t]{2}{*}{50055330} & Río Bairoa at Barrio Bairoa & $5 / 11 / 98$ & 1300 & 240 & 330 & 2.7 & 1.2 & 3.02 & Acceptable \\
\hline & & $9 / 18 / 98$ & 1700 & 310 & 5,700 & 3.2 & -- & -. & -- \\
\hline \multirow[t]{2}{*}{50055400} & Río Bairoa near Caguas & $5 / 11 / 98$ & 1030 & $3,500 *$ & $6,100 *$ & 2.4 & -- & 5.43 & Poor \\
\hline & & $9 / 18 / 98$ & 1300 & 5,500 & 4,200 & 3.9 & -- & -. & -- \\
\hline 50055404 & $\begin{array}{l}\text { Tributary to Río Bairoa at } \\
\text { Highway } 1\end{array}$ & $5 / 11 / 98$ & 1300 & ND & ND & $\begin{array}{l}\text { Stagnant } \\
\text { water }\end{array}$ & -- & 0.70 & Not ranked \\
\hline \multirow[t]{2}{*}{50055410} & Río Bairoa at mouth & $5 / 11 / 98$ & 1140 & 350 & $30 *$ & 23 & -- & 7.52 & Fair \\
\hline & & $9 / 18 / 98$ & 1300 & 10,000 & 790 & 24 & -- & -- & -- \\
\hline \multirow[t]{2}{*}{50057500} & Lago Loíza \#4 & $5 / 25 / 98$ & 1030 & 500 & $170 *$ & $\begin{array}{l}\text { In } \\
\text { reservoir }\end{array}$ & -- & $-\bullet$ & Acceptable \\
\hline & & $7 / 24 / 98$ & 1035 & 84 & $110 *$ & $\begin{array}{l}\text { In } \\
\text { reservoir }\end{array}$ & -- & -- & -- \\
\hline \multirow[t]{2}{*}{50057800} & $\begin{array}{l}\text { Río Cañas at Barrio } \\
\text { Jagueyes }\end{array}$ & $5 / 5 / 98$ & 0850 & 64 & 300 & 0.9 & 0.5 & 2.75 & Good \\
\hline & & $11 / 19 / 98$ & 1030 & 170 & 310 & 9.2 & -- & -- & -- \\
\hline \multirow[t]{2}{*}{50058010} & $\begin{array}{l}\text { Río Cañas at Highway } 1 \text { at } \\
\text { Barrio Río Cañas }\end{array}$ & $5 / 5 / 98$ & 1040 & 1,000 & 500 & 1.1 & 0.7 & 3.60 & Acceptable \\
\hline & & $11 / 19 / 98$ & 1400 & 360 & 400 & 12 & -- & -- & -- \\
\hline \multirow[t]{2}{*}{50058300} & $\begin{array}{l}\text { Quebrada Arenas at } \\
\text { Highway } 175\end{array}$ & $5 / 6 / 98$ & 0930 & 580 & 330 & 0.8 & -- & 3.49 & Acceptable \\
\hline & & $11 / 17 / 98$ & 1230 & 1,200 & 380 & 9.3 & -- & -- & -- \\
\hline \multirow[t]{2}{*}{50058375} & $\begin{array}{l}\text { Tributary to Río Cañas at } \\
\text { Highway } 798\end{array}$ & $5 / 8 / 98$ & 1200 & 4,500 & 860 & 1.0 & -- & 1.74 & Poor \\
\hline & & $11 / 17 / 98$ & 1400 & 33,000 & 6,600 & 5.0 & -- & -- & -- \\
\hline \multirow[t]{2}{*}{50058610} & $\begin{array}{l}\text { Tributary to Lago Loíza at } \\
\text { Highway } 175\end{array}$ & $5 / 8 / 98$ & 1100 & $2,000 *$ & 870 & 0.06 & -- & 0.27 & Acceptable \\
\hline & & $11 / 17 / 98$ & 1030 & 390 & 450 & 0.6 & -- & -- & -- \\
\hline
\end{tabular}




\section{RESULTS AND INTERPRETATION}

A major assumption in the interpretation is that streamflow during low-flow conditions is derived from ground-water discharge, and that the fecal contamination during stream base-flow conditions is primarily derived from sources discharging directly into stream courses or near the riparian zone. It is also assumed that, with an average of two samples obtained at least several months apart during stream low-flow recession periods at numerous locations throughout a watershed, it is possible to define, on a qualitative basis, the relative sanitary quality at the site with respect to the other sampling locations.

Based on these assumptions, the analytical results for fecal coliform and fecal streptococcus bacteria concentrations from the 37 stream sampling stations and the Lago Loíza reservoir sampling station were used to characterize the sanitary quality of $123 \mathrm{mi}$ of perennial stream channels within the municipio of Caguas and 14 mi of stream channels outside the municipal boundaries but with drainage into the Río Grande de Loíza and the municipio of Caguas (table 5). Bacteriological data were insufficient to adequately assess the sanitary quality of approximately 26 stream miles within the municipio of Caguas.

Based on the results, a relative ranking of the stream sanitary quality was established to delimit stream channels as being either poor, fair, acceptable, or good (table 6). This relative ranking was established using the Puerto Rico Water Quality Standards for fecal coliform of 2,000 colonies per $100 \mathrm{~mL}$, and the following rationale:

Sampling stations with fecal coliform bacteria concentrations greater than 2,000 colonies per $100 \mathrm{~mL}$ for both sample dates were considered poor. The stream segment given the same classification was extended upstream and downstream as follows:

If another sampling station was established upstream and/or downstream within the same order stream and the results were comparable, the same classification was given for the entire stream segment between both sample stations; if the upstream station and/or downstream station was classified differently, the classification was extended to mid-point; and if no other sampling station was located upstream, the same classification was extended upstream not more than $0.6 \mathrm{mi}$ along the main trunk of the stream. For stream segments, an upstream distance greater than $0.6 \mathrm{mi}$ of the sampling station and for its tributaries, the same classification was assigned but using the terminology of presumed poor. If no other sampling station was established downstream, the same classification was used up to a distance of $0.6 \mathrm{mi}$ along the main channel of the stream (same stream order), with the presumed poor classification assigned downstream of the 0.6-mi distance.

Within the city of Caguas, an exception was made where the stream classification of presumed poor was extended upstream to the urban limits. This was justified because of six sampling stations established within the urban reaches of streams, five had fecal coliform concentrations ranked as poor (stations 50052860, 50054500, 50055250, 50055400, and 50058375), and one ranked as fair (50054300); and 11 of 12 samples had fecal coliform concentrations above 2,000 colonies per $100 \mathrm{~mL}$.

Sampling stations at which fecal coliform bacteria concentrations were equal to or greater than 2,000 colonies per $100 \mathrm{~mL}$ in one sample occasion, but below 2,000 colonies per $100 \mathrm{~mL}$ in the second sample run were classified as fair. The classification of presumed fair was extended upstream and downstream of the sampling station following the same rationale as stated previously for poor and presumed poor.

Sampling stations where fecal coliform bacteria concentrations were equal to or less than 2,000 colonies per $100 \mathrm{~mL}$ on both sample occasions were classified as acceptable. The classification of presumed acceptable was assigned for stream segments upstream and downstream of the sampling station using the same rationale described previously. 


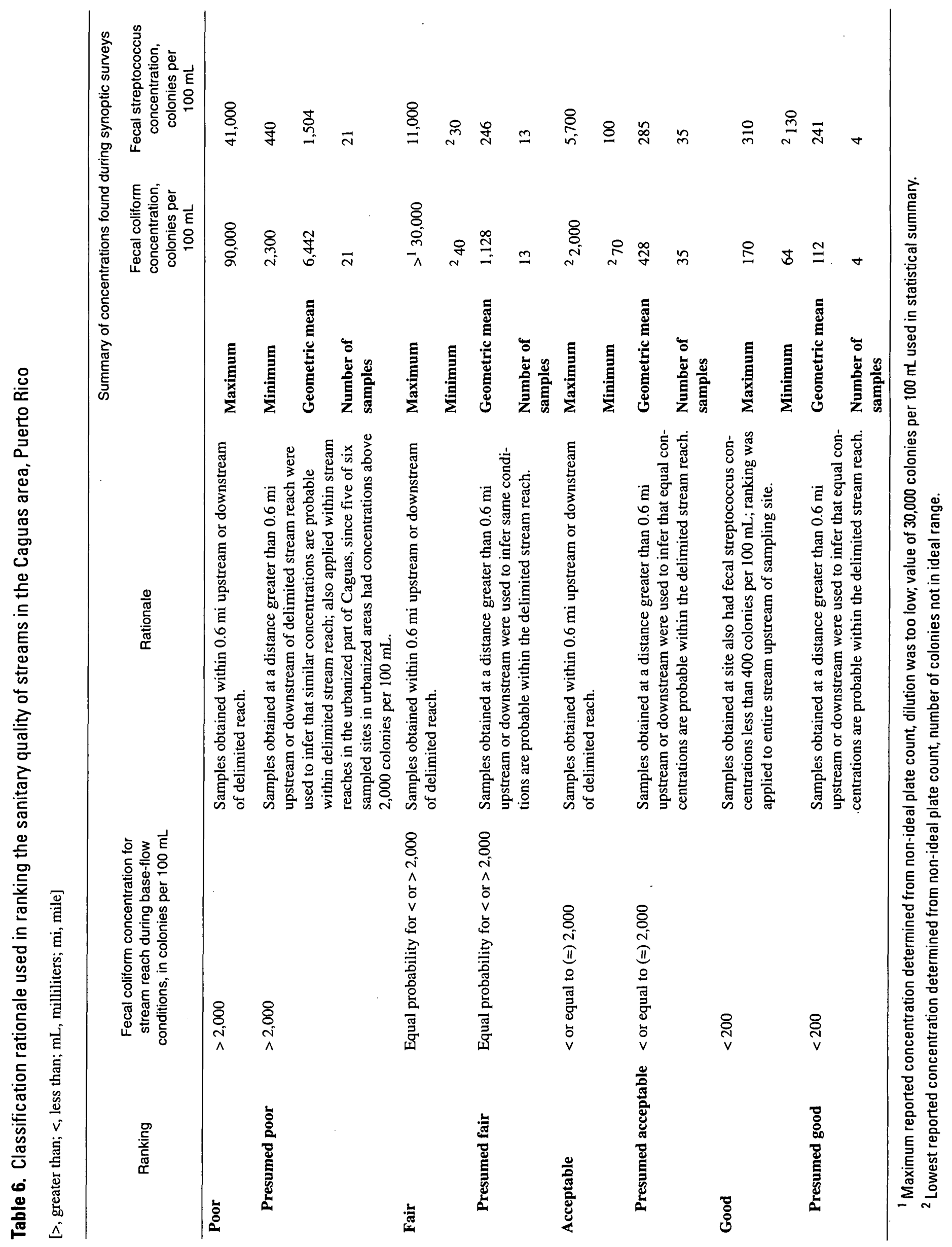


If both samples had fecal coliform bacteria concentrations below 200 colonies per $100 \mathrm{~mL}$ and fecal streptococcus concentrations below 400 colonies per $100 \mathrm{~mL}$, the good and presumed good classifications were assigned in the same manner as the other rankings. The presumed ranking was not assigned to any stream (or tributary) in which no fecal coliform and fecal streptococcus analyses were obtained, given the low probability of bacteriological concentrations being within the classification of good or presumed good (only 4 of 74 samples ( 5 percent) met the requirements for the good classification).The only sampling stations classified as having a good sanitary quality were at Quebrada Beatriz (station 50053200) and Río Cañas (station 50057800).

The presumed prefix was used in the ranking of several streams having drainage areas of less than $3 \mathrm{mi}^{2}$. At these streams, the classification was based on sampling results obtained at streams draining adjacent watersheds with similar land-use conditions as determined from field inspections and 1994 aerial photographs. The only exceptions were tributaries (or parts of tributaries) of the Río Turabo in Barrios Turabo and Tomás de Castro. In these areas, local land-use conditions vary substantially from adjacent watersheds. At Barrio Turabo, the potential effects of the municipal landfill on the headwaters of a nearby unnamed tributary to the Río Turabo is unknown; at Barrio Tomás de Castro there is a substantial difference in land use between adjacent sub-basins (among these, pasture and fallow lands undergoing change to new housing developments) to properly rank an unnamed tributary of the Río Turabo. The presumed prefix was also used at stations sampled twice, but for which only one sample was obtained during base-flow conditions. In these cases, the station (presumed) ranking was based only on the analytical results of the sample obtained at stream base-flow conditions.

No classification was made for the part of the Río Cagüitas upstream of the Caguas-Cidra municipio boundary, because bacteriological data were insufficient. The only samples obtained at stations 50055092 and 50055097 were collected during the rising stage (RS) of a runoff event.
A summary of the bacteriological results, the classification assigned at sampling stations, and stream miles classified under each ranking is presented in table 7. Results from the 38 streamsampling stations and the sampling station in the reservoir were extrapolated to classify a total of $137 \mathrm{mi}$ of stream courses including the $4.5 \mathrm{mi}$ of Lago Loíza reservoir bordering the municipio of Caguas (plate 1). Approximately 26 stream miles were not classified because of insufficient data.

It was necessary to indicate on plate 1 potential contaminant sources from unsewered rural communities in proximity to stream courses, especially along stream segments where fecal coliform concentrations were below 2,000 colonies per $100 \mathrm{~mL}$. It is possible that the indicator bacteria became stressed or injured by household waste-water discharges, rendering them incapable of growth and colony formation because of structure or metabolic changes associated with discharge of untreated wastes (American Public Health Association, 1998). Hence, an additional symbol was added to plate 1 to show potential sources of household waste-water discharges that were evident within the municipio boundary during the field surveys. Stream course segments that may be affected by these potential sources were mapped using 1994 aerial photographs. These riparian segments are potential sources of contamination and were identified as "riparian zone with potential as a source of contamination from household waste-water discharges" and delimited on plate 1. Approximately 3 stream miles were delimited with this symbol on plate 1 . Stream segments delimited with this symbol can be affected by "gray-water" discharges and septic tank effluent from housing communities bordering the riparian zones of streams or from relatively dense housing developments (generally, with one or more housing units per $1 / 5$ th-acre lot) that are located within $300 \mathrm{ft}$ of the stream courses. The adequacy of the 300 $\mathrm{ft}$ setback distance between houses with septic tanks and stream courses is unknown. Determination of adequate setback distance must consider rainfall recharge, hydrogeology, and housing density, in addition to the soil percolation rate typically used in designing septic tank systems. The $300-\mathrm{ft}$ distance, however, can be used as an initial estimate to delimit potential sources of contamination to streams from 
Table 7. Summary of the bacteriological analysis, the water-quality ranking determined for sampling stations, and stream miles classified under each ranking

[Total miles include the segment of Río Grande de Loíza and Lago Loíza within the municipal boundary and segment of stream channels outside the municipal boundary with drainage to Caguas; --, no data]

\begin{tabular}{lccccc}
\hline Classification & $\begin{array}{c}\text { Sampling } \\
\text { stations with } \\
\text { same } \\
\text { classification }\end{array}$ & $\begin{array}{c}\text { Percent of } \\
\text { total } \\
\text { sampling } \\
\text { stations } \\
\text { ranked }\end{array}$ & $\begin{array}{c}\text { Stream miles } \\
\text { with same } \\
\text { classification }\end{array}$ & $\begin{array}{c}\text { Percent of } \\
\text { total } \\
\text { stream } \\
\text { miles }\end{array}$ & $\begin{array}{c}\text { Percent of } \\
\text { total } \\
\text { stream } \\
\text { miles } \\
\text { ranked }\end{array}$ \\
\hline Poor & 10 & 28.9 & 38.2 & 23.4 & 28 \\
$\quad$ Presumed poor & 1 & -- & -- & -- & -- \\
Fair & 6 & 18.4 & 25.6 & 15.7 & 18.7 \\
$\quad$ Presumed fair & 1 & -- & -- & -- & -- \\
Acceptable & 17 & 47.4 & 59.8 & 36.7 & 43.7 \\
$\quad$ Presumed acceptable & 1 & -- & -- & -- & -- \\
Good & 2 & 5.3 & 13.1 & 8.0 & 9.6 \\
$\quad$ Presumed good & 0 & -- & -- & -- & -- \\
Not classified & 3 & -- & 26.4 & 16.2 & -- \\
\hline
\end{tabular}

unsewered communities, because viruses can move as far as $215 \mathrm{ft}$ from a septic tank in sandy soils (Vaughn and others, 1983) and persist up to 131 days in ground water (Stramer, 1984).

Grazing dairy and beef cattle may also be a major source of fecal contamination in several streams, since cattle are not restricted from wandering into stream courses. According to the 1997 agricultural census (U.S. Department of Commerce, 1998b), there were an estimated 859 head of dairy cattle and 1,135 head of beef cattle in the municipio of Caguas. The number of dairy cattle has declined substantially since the agricultural census of 1992 when 2,707 head of dairy cattle were counted (U.S. Department of Commerce, 1994).
Dairy and beef production are primarily concentrated within the drainage basins of the Quebrada de las Quebradillas, Río Bairoa, and Río Caguiitas. The sanitary quality of Quebrada Las Bambúas was classified as poor and may be related to the hog and poultry enterprises that are concentrated in this drainage basin. In the 1997 agricultural census, there were an estimated 3,000 hogs and pigs (as compared to 6,602 in the 1992 agricultural census) and 399,000 poultry (as compared to 321,992 in the 1992 agricultural census) in the municipio of Caguas. 
30 Surface-Water, Water-Quality, and Ground-Water Assessment of the Municipio of Caguas, Puerto Rico, 1997-99 


\title{
CHAPTER C:
}

\section{The Hydrogeologic Terranes and Ground-Water Resources in the Municipio of Caguas, Puerto Rico, 1997-99}

\author{
By Jesús Rodríguez-Martínez, Fernando Gómez-Gómez, and Mario L. Oliveras-Feliciano
}

\section{PURPOSE AND SCOPE}

The USGS in cooperation with the municipio of Caguas conducted an investigation of the surface- and ground-water resources of the municipio area (fig. 1 in plate 2). As part of this study, the municipio of Caguas was differentiated into areas with similar hydrogeologic characteristics, referred to as hydrogeologic terranes. Geologic, topographic, soil, hydrogeologic, and streamflow data were used to delineate the various hydrogeologic terranes. These data were obtained from field reconnaissance, USGS topographic and geologic maps, collected streamflow data, and available published information. Some of the hydrogeologic terranes extend beyond the boundaries of the municipio of Caguas. However, because the scope of this study is limited to the municipio of Caguas, only the areal extent of the hydrogeologic terranes within this municipio is presented in this report.

The information compiled and generated during this study will help the municipio of Caguas in developing its land and water resources in a sustainable manner, and provide hydrogeologic information to the general public in a format that can be easily understood and utilized.

\section{METHODOLOGY}

The factors used to distinguish the municipio of Caguas into hydrogeologic terranes were, in order of importance, (a) the underlying rock and sediment type, (b) the soil thickness and infiltration capacity, (c) the ground-water flow rate, (d) the general depth to water table, and (e) the land-surface slope. The geologic substrata and associated attributes, such as the presence of fractures, joints, and stratification, were determined from USGS geologic maps and lineamenttrace analyses (Rogers, 1979; Broedel, 1961; Pease, 1968; Glover, 1982; Sander and others, 1997). The soil thickness and infiltration capacity were obtained from soil maps published by the Soil Conservation Service (U.S. Department of Agriculture, 1976). The groundwater flow rate was estimated from stream low-flow measurements and using the Q-98 and Q-90 flows (streamflows equaled or exceeded 98 and 90 percent of the time, respectively). The general depth to ground water was obtained from USGS files and field surveys. The land-surface slope was obtained by computer processing a digitized topographic map of Caguas. The hydrogeologic terranes are delineated in a 1:30,000-scale map of the municipio of Caguas, which also includes drainage sub-basins (plate 2). Lithologic data from Puig and Rodríguez (1993), in conjunction with ground-water level data obtained during this study, were used to construct a hydrogeologic section along the Caguas alluvial valley (fig. 3 in plate 2). The ground-water level data also were used to define the potentiometric surface in the Caguas alluvial valley (plate 2). Water samples collected from five private water-supply wells located in hydrogeologic terranes underlain by igneous rocks were analyzed for major dissolved constituents (table 8).

Some of the factors mentioned above, although considered separately in this report, are generally interrelated, the nature of a particular factor being dependent on the nature of one or more other factors. For example, geology and land-surface slope are interrelated and, at the same time, highly affected by the type and thickness of the soil cover present. Similarly, geology and land-surface slope control, to a large degree, the ground-water flow rate. Low flows, an indirect measure of the ground-water flow or 


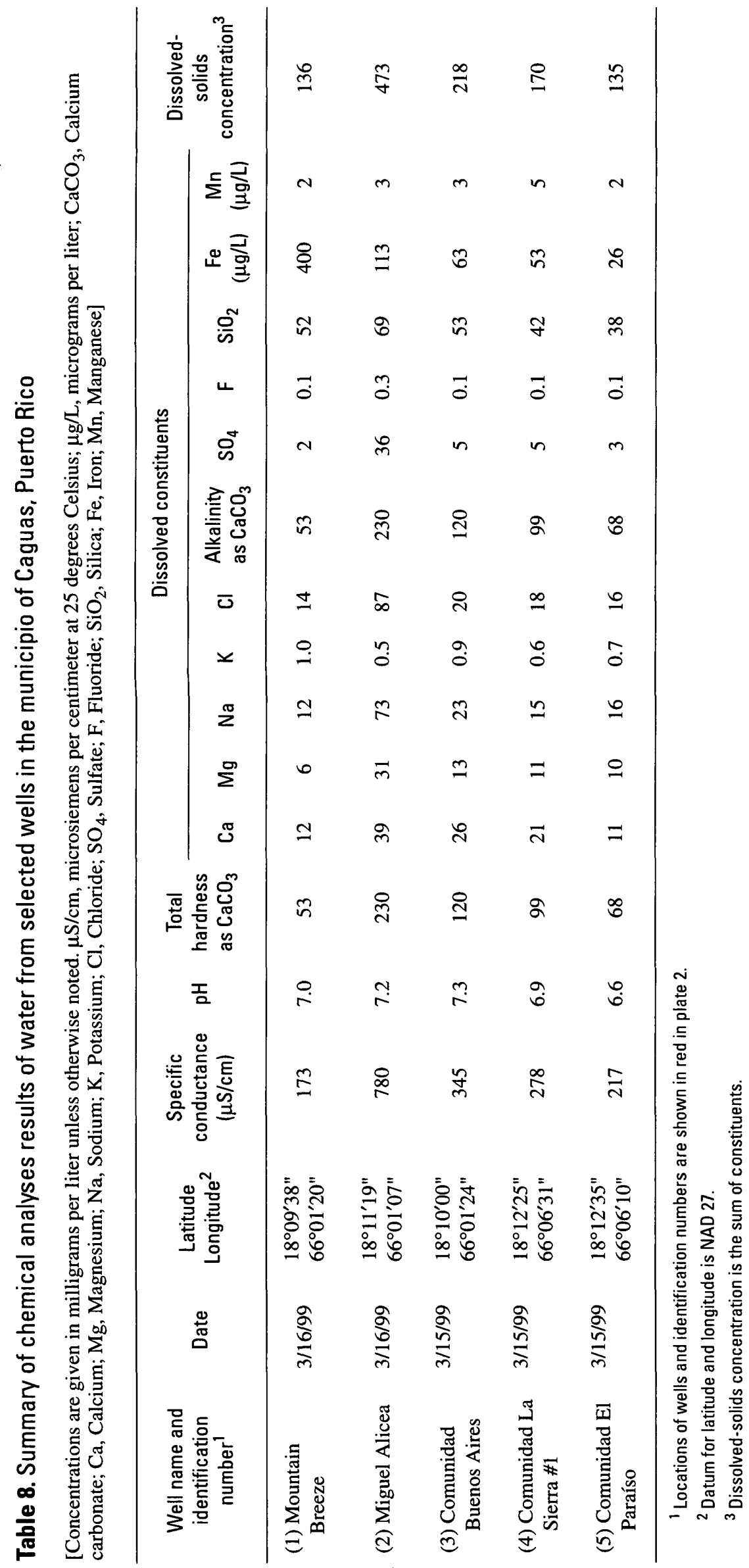


equivalent effective recharge in the aquifer(s) within a drainage basin, are highly dependent in geologic factors such as type of lithology, stratification, and the presence of fractures and fissures.

Lineament-trace analysis is used to identify land-surface features such as topographic and straight drainage features, geologic contacts, and tonal differences in soil colors to indicate subsurface fractures that may be zones of enhanced ground-water flow in an otherwise low-permeability formation (Lattman, 1958). For this study, two independent observers identified lineament traces by analyzing aerial photos. Those lineaments recognized by both observers, as suggested by Sander and others (1997), were considered to have the highest hydrogeologic importance. These features were corroborated in the field and are presented in plate 2.

Stream low-flow values can be used to approximate the ground-water flow rate in a corresponding drainage basin, and therefore, can be used to infer the ground-water development potential of a particular area (Berg and others, 1984; Farvolden and Nunan, 1970; Ineson and Downing, 1964). This equivalence or approximation of low flows to groundwater flow rate assumes that (a) ground water in a particular ground-water basin discharges entirely into the stream and its tributaries, and (b) ground-water discharge into the stream and tributaries is restricted to aquifer(s) in the corresponding drainage basin. This approximation also assumes that the contributing ground-water catchment does not vary seasonally in extent, due to fluctuating ground-water levels. Lowflow measurements were preferentially made at stream junctions, and wherever possible at stream sections coinciding with the boundaries of different geologic units as delineated on the 1:20,000-scale geologic quadrangle maps. This discharge-measurement strategy provided the low-flow values required to calculate the contribution of ground-water flow from the drainage area of each of the measurement points. The measured low-flow values were normalized by dividing the discharge (measured in cubic feet per second and converted into gallons per day) by drainage basin area (expressed in square miles) to remove the effect of drainage basin area, and thus are expressed as gallons per day per square mile $\left(\mathrm{gal} / \mathrm{d}-\mathrm{mi}^{2}\right)$ and in the equivalent effective recharge in inches per year (in/yr).
Pérez-Blair (1997) used the Q-98 flow (streamflow value equaled or exceeded 98 percent of the time) as an indicator of ground-water discharge into a stream to provide a good approximation of the ground-water flow in the corresponding drainage basin. Berg and others (1997) used the Q-90 (streamflow value equaled or exceeded 90 percent of the time) determined from 30 years of streamflow data. For this study, the range between the Q-98 and Q-90 flows was used to approximate ground-water flow in the aquifer(s) within the corresponding drainage basin. The Q-98 and Q-90 flows were obtained using the graphical correlation method described in Chapter A. By calculating the Q-98 and Q-90 flows for various drainage sub-basins, as shown in table 9, it was possible to obtain a reasonable approximation of the ground-water flow rate in the various hydrogeologic terranes delineated in the municipio of Caguas (table 9).

Stream low-flow contributions from outside the municipio of Caguas were not considered in the analysis. That fraction of low flow from ground-water discharge originating outside the municipio of Caguas and from regional ground-water flow is not known, and consequently, cannot be accounted for in the analysis. Given the limited extent of the water-bearing units, however, and their generally low permeability, it is assumed that local ground-water flow systems predominate.

Locally, low flows (and equivalent groundwater flows) within similar hydrogeologic terranes can differ as a result of variable land cover and the resulting evapotranspiration. Typically in tropical areas, stream low flow in volcanic terranes is controlled by relatively high rainfall infiltration provided by forest cover (Bruijnzeel, 1990). Removal of forest cover and change in land use for agricultural purposes was found to result in a reduction of stream low flow from an equivalent of 6 to 2 inches (in.) of rainfall (Ramos-Ginés, 1997). The effects of evapotranspiration on shallow ground-water flow can be important during the dry season, and even with a constant land cover, variations may occur in the low flows. At present, low flows in any drainage basin reflect the prevailing precipitation pattern (effective recharge). Ground-water flow and equivalent stream low flow in any drainage basin will change as variations in effective recharge occur as a result of changes in the precipitation pattern. 
Table 9. Hydrogeologic features, including areal extent, normalized ground-water flow per square mile, effective recharge, and relation with hydrogeologic terranes, of selected sub-basins in the municipio of Caguas, Puerto Rico

$\left[\mathrm{mi}^{2}\right.$, square miles; gal/d-mi ${ }^{2}$, gallons per day per square mile; in/yr, inches per year; <, less than; CaHT, Caguas hydrogeologic terrane]

\begin{tabular}{|c|c|c|c|c|c|c|}
\hline $\begin{array}{l}\text { Sub-basin Identification } \\
\text { number and name }{ }^{1}\end{array}$ & $\begin{array}{l}\text { Area } \\
\left(\mathrm{mi}^{2}\right)\end{array}$ & $\begin{array}{l}\text { Approximate } \\
\text { normalized } \\
\text { Q-90 ground- } \\
\text { water flow } \\
\text { (gal/d-mi }{ }^{2} \text { ) }\end{array}$ & $\begin{array}{l}\text { Q-90 effective } \\
\text { recharge } \\
\text { (in/yr) }\end{array}$ & $\begin{array}{c}\text { Approximate } \\
\text { normalized Q-98 } \\
\text { ground-water } \\
\text { flow } \\
\left.\text { (gal/d-mi }{ }^{2}\right)\end{array}$ & $\begin{array}{c}\text { 0-98 } \\
\text { effective } \\
\text { recharge } \\
\text { (in/yr) }\end{array}$ & $\begin{array}{l}\text { Hydrogeologic } \\
\text { terranes that } \\
\text { occur within the } \\
\text { sub-basin }\end{array}$ \\
\hline $\begin{array}{l}\text { (1) Tributary to } \\
\text { Quebrada Naranjito at } \\
\text { Barrio Borinquen }\end{array}$ & 2.24 & $<100,000$ & $<1$ & $<100,000$ & $<1$ & $\begin{array}{l}\mathrm{CaHT} 3, \mathrm{CaHT} 2 \text {, } \\
\text { and CaHT1 }\end{array}$ \\
\hline $\begin{array}{l}\text { (2) Quebrada Naranjito } \\
\text { at Barrio Borinquen }\end{array}$ & 4.37 & 259,000 & 5 & 129,600 & 3 & CaHT2 \\
\hline $\begin{array}{l}\text { (3) Quebrada Maracai at } \\
\text { Barrio Borinquen }\end{array}$ & 2.31 & 778,000 & 16 & 648,000 & 13 & $\begin{array}{l}\mathrm{CaHT} 4, \mathrm{CaHT} 2, \\
\mathrm{CaHT} 3, \text { and } \\
\mathrm{CaHT} 1\end{array}$ \\
\hline $\begin{array}{l}\text { (4) Río Turabo at Barrio } \\
\text { San Salvador }\end{array}$ & 1.38 & 778,000 & 17 & 648,000 & 14 & $\begin{array}{l}\mathrm{CaHT} 2, \mathrm{CaHT} 3 \text {, } \\
\text { and CaHT4 }\end{array}$ \\
\hline $\begin{array}{l}\text { (5) Quebrada Sonadora } \\
\text { at Barrio Beatriz }\end{array}$ & 1.20 & 194,000 & 3 & 115,000 & 2 & CaHT2 \\
\hline $\begin{array}{l}\text { (6) Quebrada Beatriz at } \\
\text { Barrio Beatriz }\end{array}$ & 3.19 & 130,000 & 3 & $<100,000$ & 2 & $\begin{array}{l}\mathrm{CaHT} 2 \text { and } \\
\mathrm{CaHT} 4\end{array}$ \\
\hline $\begin{array}{l}\text { (7) Quebrada de Las } \\
\text { Quebradillas at Barrio } \\
\text { Beatriz }^{3}\end{array}$ & 3.8 & 194,000 & 4 & 130,000 & 3 & $\mathrm{CaHT} 2$ \\
\hline $\begin{array}{l}\text { (8) Quebrada de Las } \\
\text { Quebradillas above } \\
\text { Dam at Barrio Turabo }\end{array}$ & 2.42 & 259,000 & 5 & 130,000 & 3 & CaHT2 \\
\hline $\begin{array}{l}\text { (9) Río Cañaboncito at } \\
\text { Barrio Cañaboncito }\end{array}$ & 1.6 & 122,000 & 2.5 & 81,000 & 1.7 & $\mathrm{CaHT} 2$ \\
\hline
\end{tabular}

${ }^{1}$ Sub-basin identification number shown in gray in plate 2.

${ }^{2}$ Hydrogeologic terranes are in order of decreasing areal extent within the sub-basin.

${ }^{3}$ Drainage basin extends beyond the borders of the municipio of Caguas. 
The ground-water flow estimates included lowflow measurements from drainage sub-basins of variable size. Even though ground-water flow estimates were normalized on a unit area basis for comparison between hydrogeologic terranes, it is possible that the size of the drainage sub-basins could have some effect on the low-flow values used in the analysis. For example, as the size of the basin increases, the distribution of elements that determine ground-water transmissivity and storage (primary porosity, fractures, joints, bedding planes, thickness of the weathered zone), and climatological factors (precipitation, evapotranspiration) become increasingly heterogeneous. Similarly, land cover may vary within larger basins. This departure from uniformity in these factors may result in an uneven generation of low flow across the basin. Thus, measured low flows may only partially represent the spatial variation of ground-water flow in the aquifer(s). This effect, after normalization, is referred to as the residual effect (Farvolden and Nunan, 1970). The residual effect seems to be important in basins covering tens to hundreds of square miles. Because the basins in the current study do not exceed $10 \mathrm{mi}^{2}$ in area, the residual effect may be minimal or none.

\section{RESULTS AND INTERPRETATION}

The municipio of Caguas can be differentiated into four hydrogeologic terranes (CaHT). These are delineated on plate 2 and described below in descending order of ground-water development potential.

CaHT1-This hydrogeologic terrane consists of Quaternary alluvium and Quaternary terrace deposits, and may include the underlying weathered zone of the igneous basement. The alluvium and terrace deposits are composed mostly of silt, clay, and fine sand, with subordinate amounts of coarse sand and gravel. The Quaternary alluvium deposits are mostly restricted to narrow bands along the streams. CaHT1 is restricted to lowlands, with a slope not exceeding 15 degrees, and includes the lower reaches of the main streams of the municipio of Caguas. The soil cover thickness ranges from $4 \mathrm{ft}$ in the Quaternary alluvium to $7 \mathrm{ft}$ in the Quaternary terrace deposits. The infiltration capacity of the soil cover ranges from 0.05 to $0.5 \mathrm{ft}$ per hour ( $\mathrm{ft} / \mathrm{hr}$ ) in the Quaternary alluvium, and from 0.05 to $0.2 \mathrm{ft} / \mathrm{hr}$ in the Quaternary terrace deposits. The Quaternary alluvium and Quaternary terrace deposits have a reported thickness that ranges from 20 to $180 \mathrm{ft}$, but averages about $80 \mathrm{ft}$ (Puig and Rodríguez, 1993). The average transmissivity value for CaHT1 is about 300 square feet per day $\left(\mathrm{ft}^{2} / \mathrm{d}\right)$ with reported values ranging mostly from 100 to $1,000 \mathrm{ft}^{2} / \mathrm{d}$. Transmissivity values ranging between 2,000 and $3,000 \mathrm{ft}^{2} / \mathrm{d}$ have been reported in several wells in the northern part of the hydrogeologic terrane, within the lower reaches of Río Bairoa. This high transmissivity is attributed to the Río Bairoa alluvial deposits ranging in thickness from 80 to $120 \mathrm{ft}$, which are the thickest in this hydrogeologic terrane (Puig and Rodríguez, 1993). Some of these reported localized high transmissivity values may also be the result of recharge boundary effects of nearby streams on aquifer tests. Sustained well yields in CaHT1 range mostly from 20 to 100 gallons per minute (gal/min). Localized sustained well yields greater than $100 \mathrm{gal} / \mathrm{min}$ near Río Bairoa may be the result of above-normal transmissivity and induced flow from the adjacent river. In general, yields higher than $100 \mathrm{gal} / \mathrm{min}$ are generally the result of induced seepage from nearby streams and intersecting locally enhanced fracture zones that, in turn, are hydraulically connected to a recharge source such as a stream.

A synoptic survey of water levels at wells made on August 11, 1999, indicated levels ranged from 10 to about $40 \mathrm{ft}$ below land surface. The potentiometric map constructed for CaHT1 (plate 2) indicates that, in general, the aquifer discharges into Río Caguiitas and Río Turabo. A similar result was obtained by Puig and Rodríguez (1993) in a synoptic seepage survey conducted on March 25, 1988. The potentiometricsurface contours (plate 2) can be used to infer the direction of ground-water flow, recharge sources to the alluvial and terrace deposits (hydrogeologic terrane CaHT1), and points of discharge.

CaHT2-This hydrogeologic terrane consists mainly of volcaniclastics, breccia, sandstone, siltstone with minor limestone lenses, and subordinate amounts of ash to lapilli tuff. In the vicinity of streams, CaHT2 may be hydraulically connected with small units of 
alluvium of CaHT1. The slope is variable. The thickness of soil cover varies from less than 2 to about $5 \mathrm{ft}$. The infiltration capacity of the soil cover generally ranges from 0.05 to $0.2 \mathrm{ft} / \mathrm{hr}$. The stratification varies from poorly to well developed. Fracturing does not seem to be pervasive but locally may be important. Representative sustained well yields in CaHT2 are mostly in the range of 20 to $40 \mathrm{gal} / \mathrm{min}$. Induced seepage may partially or completely sustain well yields in the vicinity of streams. Induced seepage from nearby streams and the intersection of locally thickened weathering zones related to fracturing may increase sustained yields well over $40 \mathrm{gal} / \mathrm{min}$. Specific capacity values in CaHT2 range from 1 to 2 gallons per minute per foot of drawdown (gal/min-ft), although the average value is about $1 \mathrm{gal} / \mathrm{min}$ - $\mathrm{ft}$. The depth to water table is highly variable, ranging from about 18 to $60 \mathrm{ft}$ below land surface. Estimated ground-water flow in drainage basins composed entirely of CaHT2 ranges from 115,000 to $259,000 \mathrm{gal} / \mathrm{d}-\mathrm{mi}^{2}$, which is equivalent to an effective recharge ranging from 3 to $5 \mathrm{in} / \mathrm{yr}$ (table 9). Values ranging from 648,000 to $777,000 \mathrm{gal} / \mathrm{d}-\mathrm{mi}^{2}$, or an equivalent effective recharge from 14 to $17 \mathrm{in} / \mathrm{yr}$, were estimated for the Río Turabo drainage sub-basin (plate 2), where CaHT2 is the most extensive hydrogeologic terrane. Fracture flow, however, seems to be very important in this sub-basin, affecting CaHT2 and the other hydrogeologic terranes within this drainage sub-basin. Field reconnaissance in the Río Turabo drainage sub-basin revealed the occurrence of pervasive fracturing, and more importantly, a high density of fracture intersections that substantially enhance aquifer transmissivity.

CaHT3-This hydrogeologic terrane consists predominantly of ash to lapilli-sized tuff with subordinate and scarce, thin sequences of siltstone, sandstone, and breccia. These ash and tuff units are mostly massive- to thick-bedded. Like CaHT2, this hydrogeologic terrane, may be hydraulically connected in the vicinity of streams with small alluvium units that are part of CaHT1. The topographic slope is variable. The clayey soil cover generally ranges from 1.5 to $2.5 \mathrm{ft}$ in thickness. The infiltration capacity is in the range of 0.1 to $0.2 \mathrm{ft} / \mathrm{hr}$. The few wells in CaHT3 have reported sustained yields ranging mostly from 5 to $20 \mathrm{gal} / \mathrm{min}$; however, the average sustained yield to wells is about $10 \mathrm{gal} / \mathrm{min}$. Known depths to ground water range from 20 to $55 \mathrm{ft}$ below land surface. The occurrence of artesian heads at some wells suggest local fracture flow. A ground-water flow of less than $100,000 \mathrm{gal} / \mathrm{d}-\mathrm{mi}^{2}$ and an equivalent effective recharge of less than $1 \mathrm{in} / \mathrm{yr}$ was estimated for the basin of a tributary to Quebrada Naranjito at Barrio Borinquen, where CaHT3 is the major component (table 9). Induced seepage may be important to the yields of active wells in the vicinity of streams.

CaHT4-This hydrogeologic terrane consists of igneous intrusive rocks. The topographic slope is variable. The soil cover is variable but generally ranges from 2 to $5 \mathrm{ft}$. Infiltration generally varies from 0.1 to $0.5 \mathrm{ft} / \mathrm{hr}$. A cap layer of weathered material with varying thickness is normally the main water-bearing unit in CaHT4. Except in the drainage basin of Quebrada Maracai in the southern part of the municipio of Caguas, there are few fractures and joints found in CaHT4. Ground-water flow ranging from 648,000 to $778,000 \mathrm{gal} / \mathrm{d}-\mathrm{mi}^{2}$, equivalent to an effective recharge ranging from 13 to $16 \mathrm{in} / \mathrm{yr}$, was estimated for the drainage basin of Quebrada Maracai in Barrio San Salvador. This value may be related to the substantial occurrence of fracture flow in CaHT4, as well as the other hydrogeologic terranes within this drainage sub-basin. Unless fractured, CaHT4 is not a substantial source of water to wells. Elsewhere in CaHT4, the sustained yields to the few wells drilled do not exceed $10 \mathrm{gal} / \mathrm{min}$. Comparison of similar hydrogeologic terranes with similar well yield and stream-gaging records indicate that ground-water flow in CaHT4 is below $100,000 \mathrm{gal} / \mathrm{d}-\mathrm{mi}^{2}$, which is equivalent to an effective recharge of less than $1 \mathrm{in} / \mathrm{yr}$.

The ground-water development potential in those hydrogeologic terranes, other than CaHT1, seems to be highest in those areas where the slope generally does not exceed 15 degrees and where the substrata are predominantly composed of moderately to well-stratified volcanic sandstones and breccias. Soil characteristics (type, thickness, and infiltration capacity), although highly variable, do not seem to be a major factor in ground-water development potential where they reflect both the topographic slope and the type of geologic substratum. It seems that the presence 
of fractures and joints, independent of the substrata, may locally enhance the ground-water development potential. These fractures enhance the storage and transmissive properties of the geologic bedrock. In general, the ground-water development potential substantially decreases as the topographic slope exceeds 15 degrees. This is particularly true in areas with fine-grained, massive and poorly stratified volcaniclastics, lavas, and intrusive rocks not affected by fracturing or jointing. Induced seepage from nearby streams may sustain either completely or partially the yields of pumping wells.

The ground-water development potential of CaHT1 is significantly limited by the high silt and clay content of the Quaternary alluvium and Quaternary terrace deposits. CaHT1, however, has been the most intensely developed ground-water source in the municipio of Caguas, so there is limited potential for additional development. This small potential for ground-water development in CaHT1 is further hindered by loss of recharge potential resulting from the urban expansion in the alluvial valleys of Río Cagüitas and Río Turabo. Wells in CaHT1, particularly those sited in Quaternary alluvium deposits, are susceptible to fecal coliform contamination due to induced seepage from contaminated streams such as Río Cagüitas (Díaz and others, 1995, 1996, 1997, 1998). Concentrations of manganese and iron at some wells in this hydrogeologic terrane (CaHT1) exceed the U.S. Environmental Protection Agency (USEPA) limits for drinking water (Puig and Rodríguez, 1993).

Most of the additional ground-water development potential in CaHT1 is limited to increased streamflow interception by wells, particularly in the alluvium along the Río Grande de Loíza. The streamflow interception by wells will be more effective where aquifer discharge as seepage to streams is known to occur. A temporary increase in ground-water withdrawal from CaHT1, which could exceed the net recharge and cause a partial depletion of aquifer storage, can be considered a feasible shortterm water-supply source during periods of belownormal precipitation when the water demand cannot be satisfied by existing ground-water and surfacewater supply sources. This temporary loss in aquifer storage can eventually be replenished with the return of normal precipitation. This was the case during the drought of 1994, when an increase in ground-water withdrawals of $0.5 \mathrm{Mgal} / \mathrm{d}$ to help satisfy the water demand caused a lowering in the water table in the alluvial aquifer of about $3 \mathrm{ft}$. An undetermined but likely substantial portion of the additional groundwater withdrawals during this drought, was supplied by flow from nearby streams that was induced by pumping wells.

The difference in the sustained well yields of CaHT2, CaHT3, and CaHT4 may be explained by the varying degree in stratification and grain size in the substrata of the three hydrogeologic terranes. The substrata of CaHT2 is generally the most stratified and coarse textured among the three hydrogeologic terranes. Consequently, CaHT2 has the highest ground-water development potential, following $\mathrm{CaHT} 1$. As in CaHT1, temporary depletion of aquifer storage in CaHT2 may help alleviate crises in the water supply caused by below-normal precipitation. A rational pumping strategy, however, based on the partial depletion of aquifer storage requires information on the temporal and spatial variation of recharge. Also, more detailed information is needed regarding the storage and transmissive properties of CaHT2 to properly assess the feasibility of aquifer storage depletion. The ground-water development potential of CaHT3 and CaHT4 is minimal due to the general massive or poorly stratified and predominant fine-grained nature of the geologic substrata. A reasonable ground-water development potential in these hydrogeologic terranes may exist in those areas with fractures hydraulically connected with recharge sources such as streams.

The very high ground-water flow in the upper portions of the drainage basins of Río Turabo and Quebrada Maracai in the southern extreme of the municipio of Caguas and assigned to CaHT2 and CaHT4, respectively, may be ascribed to the high density of fractures (intersection of fractures), and also to the presence of a well-developed forest cover on steep topographic slopes. The high density of fractures may have been the result of the stresses generated by the intrusion of the San Lorenzo batholith and associated satellite-intrusive plugs 
intruding into pre-existing rocks. Considering the extent and density of fractures in these two areas, the possibility exists that the ground-water recharge zones extend beyond the surface-water drainage basins. These areas are not suitable for wells because of the steep slopes, which could make well drilling expensive. In addition, by not developing groundwater resources in this area, stream low flows can be maintained at current levels.

The concentrations of the major constituents from water samples collected at five wells in hydrogeologic terranes constituted of igneous rocks are within the recommended USEPA secondary drinking water standards (table 8). Concentrations of manganese and iron, however, exceed USEPA secondary standards for drinking water at some wells in CaHT1 that penetrate alluvial valley deposits (Puig and Rodríguez, 1993). The results of the sanitary quality survey of surface waters in the municipio of Caguas (Chapter B) indicate that wells tapping the substrata near streams are highly susceptible to fecal bacteria contamination.

\section{SUMMARY AND CONCLUSIONS}

To meet the need for an adequate supply of safe drinking water, the USGS in cooperation with the Office of the Mayor of the Municipio Autónomo de Caguas, conducted an integrated surface-water, waterquality, and ground-water assessment of the municipio of Caguas. Because the supply of safe drinking water is critical during dry periods, the surface-water assessment focused on low-flow characteristics of rivers in the municipio of Caguas.

\section{Low-flow and flow-duration characteristics} were evaluated at 4 continuous-record gaging stations and 16 partial-record stations (tables 1,2). Of the 4 continuous monitoring stations, 1 is located along Río Grande de Loíza, 2 along Río Cagüitas, and 1 along Río Turabo, in which Ríos Cagüitas and Turabo are second-order tributaries of Río Grande de Loíza. The 16 partial-record stations are distributed among a number of first- to fourth-order streams in the Río Grande de Loíza drainage basin (plate 1). Two of these stations contributed less than $0.1 \mathrm{ft}^{3} / \mathrm{s}$ each, based on the 99 percent of time discharge. Two drainage basins, Río Turabo and Quebrada Maracai at Barrio San
Salvador are basins in which the minimum streamflow study indicates a relatively high discharge per unit drainage area. The surface-water contribution of these two basins is $3.4 \mathrm{ft}^{3} / \mathrm{s}$, at a low-flow discharge that is met or exceeded 99 percent of the time.

In addition to low-flow characteristics in the surface-water assessment, important surface-water resource information also was compiled, including locations of potential reservoir stations, flood-hazard areas, water-supply filtration plants, waste-water treatment plants, drainage-basin boundaries, and stream-gaging station locations (plate 1). The stream low-flow statistics presented in this report document the general hydrology under current land and water uses. Low-flow statistics may significantly change as a result of streamflow diversions for public supply, increase in ground-water development, waste-water discharges and flood-control measures. As a result, the current analysis will provide baseline information to determine impacts and to develop water budgets.

For example, during the period of this waterresources assessment, several major hydrologic modifications affected the flow of surface water in the Caguas area. Among these were new public watersupply intakes for the Caguas Norte and San Antonio filtration plants, which will result in an increase in the public supply at Caguas, but a decrease in the firm yield from Lago Loíza to the downstream communities served from this source. Additionally, the Río Turabo was being channelized for flood-control purposes and the left bank levee, when completed, may isolate the pump station intake for the Quebrada de las Quebradillas (Caguas) filtration plant from this source. This would result in a lowering of its safe yield to the Q-99 flow duration of Quebrada Beatriz, which was estimated at $0.5 \mathrm{ft}^{3} / \mathrm{s}(0.32 \mathrm{Mgal} / \mathrm{d})$ as compared to a Q-99 streamflow duration at the original pump-station intake on the Río Turabo of $4.7 \mathrm{ft}^{3} / \mathrm{s}(3.04 \mathrm{Mgal} / \mathrm{d})$.

All perennial streams in the municipio of Caguas, including water contained by the Carraízo dam in Lago Loíza, are classified as Class SD waters on the basis of their designated use. This classification applies to surface waters intended for use (or with the potential for use) as a raw water source of public supply, propagation and preservation of desirable 
aquatic species, as well as primary and secondary contact recreation. With the exception of surface waters used for primary contact recreation, the sanitary quality standard (goal) for Class SD waters in Puerto Rico is primarily based on fecal coliform concentrations (Junta de Calidad Ambiental de Puerto Rico, 1990). To meet the sanitary quality standard, the fecal coliform geometric mean concentration obtained from five samples collected in sequential order should not exceed 2,000 colonies per $100 \mathrm{~mL}$, and not more than one in five should exceed a concentration of 4,000 colonies per $100 \mathrm{~mL}$.

The results of long-term monitoring of bacterial concentrations of surface water at three stream stations (plate 1) indicate that the sanitary quality of streams in the municipio of Caguas is generally poor and has remained relatively unchanged for over a decade. A sanitary quality survey was conducted during base-flow conditions primarily to define fecal waste contamination from sources that discharge directly to or in the vicinity of stream courses. A qualitative classification method was developed and was primarily based on the Puerto Rico fecal coliform concentration goal established for Class SD-designated use surface waters, and on the concentration of fecal coliform as an indicator of fecal contamination (Junta de Calidad Ambiental de Puerto Rico, 1990). The method was applied to rank the sanitary quality at sample stations where at least two samples were obtained during stream base-flow conditions. The geometric mean of fecal coliform and fecal streptococcus concentrations (in number of colonies per $100 \mathrm{~mL}$ ) for each of the established rankings were as follows: poor - 6,442 and 1,504; fair - 1,128 and 246; acceptable - 428 and 285; and good -112 and 241 , respectively.

Because only 38 sampling stations were used to delimit the sanitary quality of approximately 163 stream miles, it was necessary to develop a rationale to extrapolate station rankings. This was done by using the prefix presumed before each of the rankings, since the bacteriological concentrations at various sampling stations along a given stream reach was significantly different at distances of as little as $0.6 \mathrm{mi}$. In summary, 137 of the 163 stream miles with drainage to or within the municipio of Caguas were ranked as follows: $38.2 \mathrm{mi}$, poor; $25.6 \mathrm{mi}$, fair; $59.8 \mathrm{mi}$, acceptable; and $13.1 \mathrm{mi}$, good. Of the 26.4 stream miles not ranked, 22.4 stream miles were outside the municipal boundary and within the Río Caguiitas and Río Bairoa drainage basins.

Based on more than 14 years of field observations and hydrologic analysis in the Caguas area, it may be inferred that the stream courses classified as poor (or presumed poor) are contaminated from continuous fecal sources of contamination, and those classified as fair (or presumed fair) and acceptable (or presumed acceptable) from intermittent sources. In urbanized areas, potential sources of fecal contamination include illegal discharge of sewage to storm-water drains especially within the older sectors of the city of Caguas; overflows from sewer mains into the stormwater drains as a result of malfunctioning sanitary sewer ejectors or clogged mains; ruptured sewer mains; and leakage from sewer mains into the local aquifer. In rural areas, potential sources of fecal contamination are gray-water discharges (gray water includes all waste water except sanitary wastes) from residential and commercial establishments along stream channels; septic tank leakage or overflows; feces contamination directly into streams by unfenced livestock; and runoff from restrained livestock pens near stream courses.

The stream segments with the greatest potential for development as surface- and ground-water sources are those classified as good or acceptable. If, however, upstream of these stream segments there are zones classified as "riparian zone with potential as a source of contamination from household waste-water discharges," then development of ground-water supplies in aquifers adjacent to these streams should not be encouraged without (a) more detailed analysis of bacteriological conditions to define diurnal variations and the application of more sensitive microbiological determinations, such as recovery enhancement tests for fecal coliform and fecal streptococcus bacteria, and (b) more in-depth evaluation of the bacteriological attenuation capacity of the ground-water bearing units. Also, a more rigorous surface-water monitoring program, including fecal coliform, fecal streptococcus, and other indicator bacteria should be required to define the variability and sources of contamination in order to implement corrective measures. 
The municipio of Caguas was separated into four principal hydrogeologic terranes based on geologic, topographic, soil, hydrogeologic, and streamflow data. The CaHT1 hydrogeologic terrane consists of waterbearing alluvium and terrace deposits of Quaternary age. Other hydrogeologic terranes (CaHT2, CaHT3, and CaHT4) consist of igneous rocks including nonstratified, fine- and coarse-grained volcaniclastics, intrusive rocks, and lava. Limestones are minor constituents of the CaHT2, CaHT3, and CaHT4 hydrogeologic terranes.

The alluvial and terrace deposits of CaHT1 typically do not develop slopes greater than 15 degrees, and are generally restricted to low-lying areas. Restricted deposits of CaHT1 alluvium are hydraulically connected with the CaHT2 hydrogeologic terrane. The CaHT2 comprises poorly- to wellstratified volcanic sandstone, breccia, and minor siltstone, ash and lapilli tuff deposits that develop variable slopes. Springs along streams in the CaHT2 hydrogeologic terrane are probably associated with water-bearing fractures. The CaHT3 hydrogeologic terrane underlies areas of variable slope and comprises ash to lapilli-sized tuff with subordinate thin sequences of volcanic siltstone, sandstone, and breccia. The CaHT4 hydrogeologic terrane underlies areas of variable slope and is composed of intrusive rocks with an overlying weathered-bedrock zone of varying thickness that functions as the main water-bearing unit.
Concentrations of iron and manganese in ground water locally exceed the USEPA secondary standards for drinking water. Because poor sanitary conditions are likely in most surface waters of the municipio of Caguas (Chapter B), wells completed in rocks that underlie streams are susceptible to fecal bacteria contamination. Potentiometric and stream-seepage data indicate that the CaHT1 alluvial aquifer is hydraulically connected with local streams, and therefore is particularly vulnerable to contamination.

Studies indicate that in relatively impermeable formations the maximum development of transmissivity occurs at the intersection of fractures. Consequently, when selecting potential stations for wells, preference needs to be given to areas with a high density of fractures or fracture intersections.

Lineament-trace analysis could substantially reduce the costs associated with the trial-and-error method used in drilling, particularly in hydrogeologic terrane CaHT4.

The integrated surface-water, sanitary quality, and ground-water assessment presented in this report can be used as an integral part of the territorial development plan of the municipio of Caguas and contribute to the implementation of measures for the sustainable development of surface- and ground-water resources. This systematic study also establishes baseline hydrologic information for the municipio of Caguas to monitor future changes in water availability and to evaluate the relation between land use, water use, and water availability. 


\section{REFERENCES}

American Public Health Association, American Public Works Association and Water Environment Federation, 1998, Standard methods for the examination of water and wastewater, 20th Edition: American Public Health Association, Washington, D.C., p. 9-26 to 9-27.

Berg, R.C., Kemptom, J.P., and Stecyk, A.N., 1984, Geology for planning in Boone and Winnebago Counties: Illinois State Geological Survey Circular 531, 69 p.

Berg, R.C., Keefer, D.A., Demissie, M., and Ramamurphy, G., 1997, Regional evaluation of ground-water and surface-water interactions: Preliminary method development and analysis: Illinois State Water Survey Miscellaneous Publication 181, $37 \mathrm{p}$.

Black and Veatch/Consulting Engineers, 1976, Water supply study for entire island of Puerto Rico: prepared for the U.S. Army Corps of Engineers, Jacksonville District, contract DACW17-75C-0059, 168 p.

Black and Veatch/Consulting Engineers, 1996, Consulting engineers report: Puerto Rico Aqueduct and Sewer Authority Facilities Inspections, Appendices 1-4.

Britton, L.J., and Greeson, P.E., eds., 1989, Methods for collection and analysis of aquatic, biological, and microbiological samples: Techniques of Water-Resources Investigations of the U.S. Geological Survey, Chapter A4, p. 37-50.

Broedel, C.H., 1961, Preliminary geologic map showing iron and cooper prospects in the Juncos quadrangle, Puerto Rico: U.S. Geological Survey Miscellaneous Geologic Investigations Series Map I-326, 1 sheet, scale 1:20,000.

Bruijnzeel, L.A., 1990, Hydrology of moist tropical forests and effects of conversion: a state of knowledge review report: UNESCO International Hydrological Programme, A Publication of the Humic Tropics Programme, Faculty of Earth Sciences, Free University, Amsterdam, 224 p.
Carter, R.W., and Davidian, Jacob, 1968, General procedure for gaging streams: Techniques of Water-Resources Investigations of the U.S. Geological Survey, Chapter A6, p. 6-7, 13 p.

Díaz, P.L., Aquino, Zaida, Figueroa-Álamo, Carlos, Vachier, R.J., and Sánchez, A.V., 1995, Water Resources Data for Puerto Rico and the U.S. Virgin Islands, Water Year 1994: U.S. Geological Survey Water-Data Report PR-94-1, 516 p.

Díaz, P.L., Aquino, Zaida, Figueroa-Álamo, Carlos, Vachier, R.J., and Sánchez, A.V., 1996, Water Resources Data for Puerto Rico and the U.S. Virgin Islands, Water Year 1995: U.S. Geological Survey Water-Data Report PR-95-1, 535 p.

Díaz, P.L., Aquino, Zaida, Figueroa-Álamo, Carlos, Vachier, R.J., and Sánchez, A.V., 1997, Water Resources Data for Puerto Rico and the U.S. Virgin Islands, Water Year 1996: U.S. Geological Survey Water-Data Report PR-96-1, 548 p.

Díaz, P. L., Aquino, Zaida, Figueroa-Álamo, Carlos, Vachier, R.J., and Sánchez, A.V., 1998, Water Resources Data for Puerto Rico and the U.S. Virgin Islands, Water Year 1998: U.S. Geological Survey Water-Data Report PR-98-1, 629 p.

Farvolden, R.N., and Nunan, J.P., 1970, Hydrogeologic aspects of dewatering: at Wellan. Canadian Geotechnical Journal, v. 7, p. 194-204.

Federal Emergency Management Agency, 1996, National Flood Insurance Program Q3 Flood Data: Disc 18, New Jersey, Puerto Rico, U.S. Virgin Islands, September 1996.

Glover, L. III., 1982, Preliminary geologic map of the Patillas quadrangle, Puerto Rico: U.S. Geological Survey Open-File Report 82-1097, 2 sheets, scale $1: 20,000$.

Gómez-Gómez, Fernando, 1980, Reconaissance of six solid-waste disposal sites in Puerto Rico and effects on water quality: U.S. Geological Survey Open-File Report 79-1338, 62 p.

Ineson, J., and Downing, R.A., 1964, The ground water component of river discharge and its relationship to hydrogeology: Journal of the Institute of Water Engineering, v. 18, p. 519-541. 
Interagency Advisory Committee on Water Data, 1982, Guidelines for determining flood flow frequency: Bulletin 17B of the Hydrology Subcommittee, U.S. Geological Survey, Office of Water Data Coordination, Reston, Virginia.

Junta de Calidad Ambiental de Puerto Rico, 1990, Reglamento de estándares de calidad de agua de Puerto Rico (versión enmendada, 20 de julio de 1990), 104 p.

Lattman, L.H., 1958, Technique of mapping geologic fractures and lineaments on aerial photographs: Photogrametric Engineering, v. 24, p. 568-576.

Myers, D.N., and Sylvester, M.A., 1997, Section 7.1, Fecal indicator bacteria, in Myers, D.N. and Wilde, F.D., eds., National field manual for the collection of water-quality data: Techniques of Water-Resources Investigations of the U.S. Geological Survey, Book 9, Chapter A7, 38 p.

Pease, M.H., 1968, Geologic map of the Aguas Buenas quadrangle, Puerto Rico: U.S. Geological Survey Miscellaneous Geologic Investigations Series Map I-479, 1 sheet, scale 1:20,000.

Pérez-Blair, Francisco, 1997, Ground-water resources of alluvial valleys in northeastern Puerto Rico-Río Espíritu Santo to Río Demajagua area: U.S. Geological Survey Water-Resources Investigations Report 96-4201, 25 p.

Puig, J.C., and Rodríguez, J.M., 1993, Ground-water resources of the Caguas-Juncos valley, Puerto Rico: U.S. Geological Survey Water-Resources Investigations Report 91-4079, 52 p.

Ramos-Ginés, Orlando, 1997, Water balance and quantification of total phosphorous and total nitrogen loads entering and leaving the Lago de Cidra, central Puerto Rico: U.S. Geological Survey Water-Resources Investigations Report 96-4222, $28 \mathrm{p}$.

Riggs, H.C., 1972, Low-flow investigations: Techniques of Water-Resources Investigations of the U.S. Geological Survey, Book 4, Chapter B1, $18 \mathrm{p}$.
Rogers, C.L., 1979, Geologic map of the Caguas quadrangle, Puerto Rico: U.S. Geological Survey Miscellaneous Geologic Investigations Series Map I-1152, 1 sheet, scale 1:20,000.

Sander, P., Minor, T.M., and Chesley, M.M., 1997, Ground-water exploration based on lineament analysis and reproducibility tests: Ground Water, v. 35 , no. 5 , p. $888-894$.

Searcy, J.K., 1959, Flow-duration curves: U.S. Geological Survey Water-Supply Paper 1542-A, $33 \mathrm{p}$.

Stramer, S.L., 1984, Fates of polio virus and enteric indicator bacteria during treatment in a septic tank including septage disinfection: Madison, University of Wisconsin, Ph.D. dissertation, $224 \mathrm{p}$.

U.S. Department of Agriculture, Soil Conservation Service, 1976, Soil survey of San Juan area of Puerto Rico: Sheets 24, 25, 33, and 43; scale $1: 20,000$

U. S. Department of Commerce, 1994, 1992 Census of Agriculture: Puerto Rico, Bureau of the Census, AC 92-A-52, $211 \mathrm{p}$.

U. S. Department of Commerce, 1998a, Estimates of the population of Puerto Rico municipios, July 1, 1997, and demographic components of population change: April 1, 1990 to July 1, 1997: http://www.census.gov/population/estimates/ puerto-rico.

U. S. Department of Commerce, 1998a, 1997 Census of Agriculture: http://www.nass.usda.gov/census/ census97/puertorico.

Vaughn, J.M., Landry, E.F., and Thomas, M.Z., 1983, The lateral movement of indigenous enteroviruses in a sandy sole-source aquifer, in Microbial health considerations of soil disposal of domestic wastewater: U.S. Environmental Protection Agency EPA-600/9-83-017, Washington, D.C., $402 \mathrm{p}$.

Webb, R.M.T., and Soler-López, Luis, 1997, Sedimentation history of Lago Loíza Puerto Rico, 1953-94: U.S. Geological Survey WaterResources Investigations Report 97-4108, 18 p., 9 pls. 
District Chief

Caribbean District

U.S. Geological Survey

Water Resources Division

GSA Center, Suite 400-15

651 Federal Drive

Guaynabo, Puerto Rico 00965-5703 